\title{
Monoacylglycerol Lipase Inhibition in Human and Rodent Systems Supports Clinical Evaluation of Endocannabinoid Modulators $₫$
}

\author{
Jason R. Clapper, Cassandra L. Henry, Micah J. Niphakis, Anna M. Knize, \\ Aundrea R. Coppola, Gabriel M. Simon, Nhi Ngo, Rachel A. Herbst, Dylan M. Herbst, \\ Alex W. Reed, Justin S. Cisar, Olivia D. Weber, Andreu Viader, Jessica P. Alexander, \\ Mark L. Cunningham, Todd K. Jones, lain P. Fraser, Cheryl A. Grice, R. Alan B. Ezekowitz, \\ Gary P. O'Neill, and Jacqueline L. Blankman
}

Abide Therapeutics, San Diego, California

Received July 26, 2018; accepted October 5, 2018

\begin{abstract}
Monoacylglycerol lipase (MGLL) is the primary degradative enzyme for the endocannabinoid 2-arachidonoylglycerol (2AG). The first MGLL inhibitors have recently entered clinical development for the treatment of neurologic disorders. To support this clinical path, we report the pharmacological characterization of the highly potent and selective MGLL inhibitor ABD-1970 [1,1,1,3,3,3-hexafluoropropan-2-yl 4-(2-(8-oxa3-azabicyclo[3.2.1]octan-3-yl)-4-chlorobenzyl)piperazine-1carboxylate]. We used ABD-1970 to confirm the role of MGLL in human systems and to define the relationship between MGLL target engagement, brain 2-AG concentrations, and efficacy. Because MGLL contributes to arachidonic acid metabolism in a subset of rodent tissues, we further used ABD-1970 to evaluate
\end{abstract}

\section{Introduction}

Monoacylglycerol lipase (MGLL; also known as MAG lipase, MAGL, and MGL) is a serine hydrolase enzyme that regulates the endocannabinoid and eicosanoid families of lipid signaling molecules (Grabner et al., 2017). MGLL catalyzes the hydrolysis of 2-arachidonoylglycerol (2-AG), an endogenous ligand of the cannabinoid receptors 1 and 2 ( $\mathrm{CB} 1$ and $\mathrm{CB} 2$, respectively) (Mechoulam et al., 1995; Sugiura et al., 1995), effectively terminating 2-AG signaling (Long et al., 2009a). In the nervous system, postsynaptic activity stimulates the biosynthesis and mobilization of 2-AG, which then traverses the synaptic cleft in a retrograde manner to agonize

All authors are or were formerly employees of Abide Therapeutics.

Parts of this work were previously presented in poster form at the following meeting: Clapper JR, Blankman JL, Coppola AR, Knize A, Simon G, Cisar J, Weber O, Niphakis M, Henry C, Fraser I, et al. (2016) In vivo characterization of a selective monoacylglycerol lipase inhibitor ABD-1970 in rodents and activity in models of pain (Program 617.02). Neuroscience 2016; 2016 Nov 12-16; San Diego, CA; and Blankman JL, Henry C, Knize A, Simon G, Cisar J, Weber O, Niphakis M, Clapper JR, Coppola A, Fraser I, et al. (2016) In vitro characterization of a selective monoacylglycerol lipase inhibitor ABD-1970 in human systems (Program 617.03). Neuroscience 2016; 2016 Nov 12-16; San Diego, CA.

https://doi.org/10.1124/jpet.118.252296.

S This article has supplemental material available at jpet.aspetjournals.org. whether selective MGLL inhibition would affect prostanoid production in several human assays known to be sensitive to cyclooxygenase inhibitors. ABD-1970 robustly elevated brain 2-AG content and displayed antinociceptive and antipruritic activity in a battery of rodent models $\left(E D_{50}\right.$ values of 1-2 $\mathrm{mg} / \mathrm{kg}$ ). The antinociceptive effects of ABD-1970 were potentiated when combined with analgesic standards of care and occurred without overt cannabimimetic effects. ABD-1970 also blocked 2-AG hydrolysis in human brain tissue and elevated 2-AG content in human blood without affecting stimulated prostanoid production. These findings support the clinical development of MGLL inhibitors as a differentiated mechanism to treat pain and other neurologic disorders. presynaptically localized CB1 (Katona and Freund, 2008; Kano et al., 2009). CB1 activation reduces the probability of neurotransmitter release, thus serving as a natural brake for excessive neurotransmission in active circuits (Kano et al., 2009). MGLL is colocalized with CB1 in presynaptic neurons (Gulyas et al., 2004; Katona et al., 2006) and in neighboring glia (Viader et al., 2015), positioning the enzyme to exert tight control of 2-AG signaling.

Direct pharmacological activation of the CB receptors by Cannabis preparations, tetrahydrocannabinol, and synthetic cannabinoids can elicit therapeutically beneficial effects on pain, spasticity, appetite, and nausea (Pertwee, 2012); however, dose-limiting central nervous system (CNS) effects of such exocannabinoids limit their broad use as pharmaceuticals. An alternative strategy to exocannabinoids is amplification of endocannabinoid signaling through inhibition of endocannabinoid degradation. MGLL is the major 2-AG hydrolase in the CNS and most peripheral tissues, and genetic or pharmacological inactivation of this enzyme reduces $2-\mathrm{AG}$ hydrolysis and elevates tissue $2-A G$ concentrations in rodents (Long et al., 2009b; Chanda et al., 2010; Schlosburg et al., 2010). MGLL inhibitors produce an array of CB1/2-mediated effects in animal models, including antinociceptive (Grabner et al., 2017), anxiolytic (Patel et al., 2017; Bedse et al., 2018), 
and antiepileptogenic effects (Griebel et al., 2015; von Rüden et al., 2015; Sugaya et al., 2016), without inducing the full spectrum of stereotypical behaviors observed after administration of direct CB1 agonists (Long et al., 2009b; IgnatowskaJankowska et al., 2014, 2015). These observations may reflect that MGLL inhibitors potentiate ongoing 2-AG signaling while preserving the spatial and temporal specificity of the endocannabinoid system, unlike exocannabinoids, which indiscriminately activate CB1 throughout the brain.

The product of MGLL-mediated 2-AG hydrolysis, arachidonic acid (AA), is the precursor for prostanoid signaling molecules (Funk, 2001). MGLL-mediated 2-AG hydrolysis serves as one of the sources of $\mathrm{AA}$ in the rodent nervous system (Long et al., 2009b) and contributes to brain levels of proinflammatory prostanoids (Nomura et al., 2011). MGLL inactivation in mice reduces molecular and cellular signs of neuroinflammation and is protective in models of neurodegeneration and status epilepticus through CB-independent mechanisms, suggesting involvement of prostanoid suppression (Nomura et al., 2011; Chen et al., 2012; Piro et al., 2012; Terrone et al., 2018; Zhang and Chen, 2018). The contribution of MGLL to AA metabolism in the mouse is tissue dependent (Nomura et al., 2011). This anatomic segregation suggests that inhibition of MGLL may afford some of the antiinflammatory effects associated with a reduction in prostanoid signaling without the side effects caused by nonsteroidal antiinflammatory drugs (NSAIDs) or coxibs, which reduce prostanoid signaling through the inhibition of cyclooxygenase COX1 and/or COX2 enzymes. In rodents, MGLL inhibition is devoid of gastrointestinal effects and instead protects from NSAIDinduced gastric hemorrhages via CB1 (Kinsey et al., 2011; Crowe and Kinsey, 2017). The effects of MGLL blockade on prostaglandin production and signaling in human cellular systems have, to our knowledge, not been reported but could potentially further differentiate MGLL inhibitors from COX1/2 inhibitors.

To support clinical investigation of endocannabinoid modulation, we report herein the pharmacological characterization of an advanced compound ABD-1970 [1,1,1,3,3,3hexafluoropropan-2-yl 4-(2-(8-oxa-3-azabicyclo[3.2.1]octan-3yl)-4-chlorobenzyl)piperazine-1-carboxylate] (Fig.1) (Cisar et al., 2018) that serves as a highly potent and selective MGLL inhibitor. We show that oral administration of ABD-1970 to rodents leads to potent and sustained inhibition of MGLL and accumulation of 2-AG in the brain, which is accompanied by dose-dependent antinociceptive and antipruritic activity. The antinociceptive effects of ABD-1970 combine beneficially with analgesic standards of care and occur without overt cannabimimetic effects. Studies with ABD-1970 confirm that MGLL is a principal regulator of $2-A G$ in the human brain and demonstrate that in stimulated human blood and endothelial cells, blockade of this enzyme does not affect prostanoid production and thus clearly differentiates from COX inhibition. Together, these results support the pursuit of MGLL inhibitors for the safe and differentiated treatment of neurologic disorders in humans.

\section{Materials and Methods}

\section{Drugs and Reagents}

ABD-1970 (Fig. 1) was synthesized at Abide Therapeutics (San Diego, CA), as previously described (Cisar et al., 2018). Synthesis of the potential hydrolysis product ABD-0038 [3-(5-chloro-2-(piperazin1-ylmethyl)phenyl)-8-oxa-3-azabicyclo[3.2.1]octane] (Supplemental Fig. 1) was performed at Abide Therapeutics and is described in the Supplemental Methods. The following activity-based probes were synthesized at Abide Therapeutics, as previously described: fluorophosphonate-rhodamine (FP-Rh) [2-[3-(dimethylamino)-6dimethyliminio-xanthen-9-yl]-5-[5-[10-[ethoxy(fluoro)phosphoryl] decoxycarbonylamino]pentylcarbamoyl]benzoate] (Patricelli et al., 2001), fluorophosphonate-biotin (FP-biotin) [10-[ethoxy(fluoro)phosphoryl]decyl N-[5-[5-[(3aS,4S,6aR)-2-oxo-1,3,3a,4,6,6a-hexahydrothieno[3,4-d]imidazol-4-yl]pentanoylamino]pentyl]carbamate (Jessani et al., 2005), HT-01 [ $N$-(5-(3-(5,5-difluoro-7,9-dimethyl-5H-5 $\lambda^{4}, 6 \lambda^{4}$ dipyrrolo[1,2-c:2', $1^{\prime}$-f] [1,3,2]diazaborinin-3-yl)propanamido)pentyl)$\mathrm{N}$-phenethyl-4-(4-(trifluoromethoxy)phenyl)- $1 \mathrm{H}$-1,2,3-triazole-1carboxamide] (Hsu et al., 2012), and JW912 [1,1,1,3,3,3-hexafluoropropan2-yl 4-((2-(3-(5,5-difluoro-7,9-dimethyl-5H-51 ${ }^{4}, 61^{4}$-dipyrrolo[1,2-c:2' $\left.2^{\prime} 1^{\prime}-f\right]$ [1,3,2]diazaborinin-3-yl)propanamido)ethyl)carbamoyl)piperidine-1carboxylate] (Chang et al., 2013). Other drugs and reagents were obtained as follows: Complete Freund's Adjuvant (CFA) from Chondrex Inc. (Redmond, WA), buprenorphine from China Resources Pharmaceutical Commercial Group Co. (Beijing, China), morphine from Johnson Matthey MacFarlan Smith (Edinburgh, UK), pregabalin from Sequoia (Pangbourne, UK) or Pfizer (Lyrica 50-mg capsules, lot J30252; Groton, CT), and WIN 55,212-2 [(R)-(+)-[2,3-dihydro-5methyl-3[(4-morpholinyl)methyl]pyrrolo[1,2,3-de]-1,4-benzoxazinyl](1-naphthalenyl)methanone mesylate salt] from Tocris Bioscience (Bristol, UK). Lipids, deuterated lipid standards, indomethacin, and rofecoxib were purchased from Cayman Chemical (Ann Arbor, MI). All other reagents were obtained from Sigma-Aldrich (St. Louis, MO), unless stated otherwise. For in vitro studies, drugs were dissolved in dimethylsulfoxide (DMSO). For in vivo studies, drugs were prepared daily in $0.5 \%$ methylcellulose (w/v), 7:2:1 polyethylene glycol 400/ethanol/phosphate-buffered saline (PBS) (v/v/v), $0.9 \%$ saline, or $1 \%$ Tween 80/99\% saline (v/v), as specified in the relevant Materials and Methods sections.

\begin{abstract}
ABBREVIATIONS: 2-AG, 2-arachidonoylglycerol; AA, arachidonic acid; ABD-0038, 3-(5-chloro-2-(piperazin-1-ylmethyl)phenyl)-8-oxa-3azabicyclo[3.2.1]octane; ABD-1970, 1,1,1,3,3,3-hexafluoropropan-2-yl 4-(2-(8-oxa-3-azabicyclo[3.2.1]octan-3-yl)-4-chlorobenzyl)piperazine-1-carboxylate; ABHD6, $\alpha / \beta$ hydrolase domain containing 6; ABPP, activity-based protein profiling; $\mathrm{CB}$, cannabinoid receptor; CCI, chronic constriction injury; CES, carboxylesterase; CFA, Complete Freund's Adjuvant; CNS, central nervous system; COX, cyclooxygenase; DMSO, dimethylsulfoxide; FCS, fetal calf serum; FP-biotin, fluorophosphonate-biotin or 10-[ethoxy(fluoro)phosphoryl]decyl N-[5-[5-[(3aS,4S,6aR)-2-oxo-1,3,3a,4,6,6ahexahydrothieno[3,4-d]imidazol-4-y $]$ ]pentanoylamino]pentyl]carbamate; FP-Rh, fluorophosphonate-rhodamine or 2-[3-(dimethylamino)-6dimethyliminio-xanthen-9-y ]-5-[5-[10-[ethoxy(fluoro)phosphoryl]decoxycarbonylamino]pentylcarbamoyl]benzoate; HT-01, N-(5-(3-(5,5-difluoro-7,9dimethyl-5H-5 $\lambda^{4}, 6 \lambda^{4}$-dipyrrolo[1,2-c:2', $\left.1^{\prime}-f\right][1,3,2]$ diazaborinin-3-yl)propanamido)pentyl)- $N$-phenethyl-4-(4-(trifluoromethoxy)phenyl)-1 $H$-1,2,3-triazole-1-carboxamide; HUVEC, human umbilical vein endothelial cell; IL, interleukin; JW912, 1,1,1,3,3,3-hexafluoropropan-2-y $\lambda$ 4-((2-(3-(5,5difluoro-7,9-dimethyl-5H-5 ${ }^{4}, 6 \lambda^{4}$-dipyrrolo[1,2-c:2', $\left.1^{\prime}-f\right][1,3,2]$ diazaborinin-3-y $\lambda$ )propanamido)ethyl)carbamoyl)piperidine-1-carboxylate; LC, liquid chromatography; LPS, lipopolysaccharide; MGLL, monoacylglycerol lipase (also known as MAG lipase, MAGL, and MGL); MS, mass spectrometry; MS/MS, tandem mass spectrometry; NSAID, nonsteroidal anti-inflammatory drug; PBMC, peripheral blood mononuclear cell; PBS, phosphate-buffered saline; PC3, human prostate carcinoma; PD, pharmacodynamic; $\mathrm{PGE}_{2}$, prostaglandin E2; PK, pharmacokinetic; TXB ${ }_{2}$, thromboxane B2; WIN 55,212-2, (R)-(+)-[2,3-dihydro-5-methyl-3[(4-morpholinyl)methyl]pyrrolo[1,2,3-de]-1,4-benzoxazinyl]-(1-naphthalenyl)methanone mesylate salt.
\end{abstract}




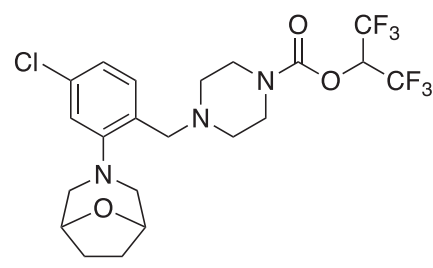

Fig. 1. Chemical structure of ABD-1970.

\section{Biochemical Studies}

Determination of Inhibitor Potency and Selectivity by Activity-Based Protein Profiling. The potency and selectivity of ABD-1970 was assessed by competitive activity-based protein profiling (ABPP) using SDS-PAGE (Simon and Cravatt, 2010) in membrane homogenates prepared from male mouse, rat, rabbit, dog, monkey, and human brain tissue; human prostate carcinoma (PC3) cells (CRL-1435, mycoplasma negative; American Type Culture Collection, Manassas, VA); and peripheral blood mononuclear cells (PBMCs) isolated from human whole blood. ABPP selectivity profiling was also performed in total homogenates prepared from human brain, liver, kidney, skin, lung, and PBMCs. The activity of the potential hydrolysis product ABD-0038 was evaluated in mouse, rat, and human brain homogenates. A detailed description of the preparation of tissue homogenates and cell lysates is provided in the Supplemental Methods.

Gel-based ABPP analysis was performed as described previously (Leung et al., 2003) using three ABPP probes: FP-Rh probe (Patricelli et al., 2001), HT-01 (Hsu et al., 2012), and JW912 (Chang et al., 2013). Brain membrane homogenates $(50 \mu \mathrm{g})$, PC3 membrane lysates $(100 \mu \mathrm{g})$, PC3 cells (100-mm dishes), human tissue homogenates $(50 \mu \mathrm{g})$, or human whole blood $(4 \mathrm{ml})$ were treated with ABD-1970 or DMSO for 30 minutes at $37^{\circ} \mathrm{C}$. Lysates were prepared from compoundtreated PC3 cells and PBMCs were isolated from compound-treated whole blood, as described in the Supplemental Methods. ABPP analysis was performed by treating the tissue or cell homogenates with $1 \mu \mathrm{M}$ FP-Rh (all tissue homogenates and PBMCs), HT-01 (rodent and dog brain homogenates), or JW912 (PC3 membranes) for 30 minutes at $25^{\circ} \mathrm{C}$. Reactions were quenched with $4 \times$ SDS-PAGE loading buffer and separated by SDS-PAGE (10\% acrylamide), and fluorescence was visualized in-gel with a ChemiDox XRS fluorescence imager (Bio-Rad, Hercules, CA). Fluorescence is shown in grayscale. Quantitation of fluorescent band intensities was performed by densitometric analysis using ImageJ software (version 1.5; National Institutes of Health, Bethesda, MD). Integrated peak intensities were generated for bands corresponding to MGLL activity (labeled with FP-Rh or JW912) or $\alpha / \beta$ hydrolase domain containing 6 (ABHD6) activity (labeled with HT-01 or JW912). IC $_{50}$ values were calculated by curve fitting semi-log-transformed data $(x$-axis) by nonlinear regression with a four-parameter, sigmoidal dose-response function (variable slope) in Prism software (version 6; GraphPad, La Jolla, CA).

Quantitative mass spectrometry (MS)-based ABPP was used to profile the selectivity of ABD-1970 in human brain homogenates using the FP-biotin probe. Human prefrontal cortex membrane homogenates were treated with $0.01-10 \mu \mathrm{M}$ ABD-1970 or DMSO for 30 minutes at $37^{\circ} \mathrm{C}$. Samples were treated with FP-biotin, enriched by avidin chromatography, and digested by trypsin, essentially as previously described (Jessani et al., 2005). Tryptic digests of the inhibitor- and DMSO-treated samples were isotopically labeled by reductive dimethylation of primary amines with natural or isotopically heavy formaldehydes (Boersema et al., 2009) and subsequently analyzed by liquid chromatography (LC)-tandem mass spectrometry (MS/MS) on a Velos Elite Orbitrap mass spectrometer (Thermo Fisher, Waltham, MA). Peptide spectral matching was performed with the complete human UniProt database using the ProLuCID algorithm (version 1.3.3, Xu et al. 2015), and the resulting matches were filtered using DTASelect (version 2.0.47, Cociorva et al. 2007). Quantification of light/heavy ratios was performed using the Cimage algorithm Weerapana et al. 2010. Data were plotted as the percent inhibition relative to DMSO-treated samples. Full details can be found in the Supplemental Methods.

Determination of Inhibitor Potency by Substrate Assays. 2AG hydrolysis activity in human brain cortex membrane homogenates (20 $\mu \mathrm{g}$ in $200 \mu \mathrm{l}$ PBS) treated with ABD-1970 or DMSO vehicle (30 minutes at $37^{\circ} \mathrm{C}$ ) was assessed using an MS-based substrate assay, essentially as previously described (Blankman et al., 2007). Full experimental details can be found in the Supplemental Methods.

The potency of ABD-1970, independent of preincubation time, was determined as the $k_{\text {inact }} / K_{\mathrm{i}}$ using purified recombinant human MGLL protein and a fluorogenic substrate assay. Full-length human MGLL was expressed in Escherichia coli bacteria with an N-terminal hexahistidine tag (Evotec, Watertown, MA), purified by nickle nitrilotriacetic acid affinity chromatography followed by size exclusion chromatography (Evotec), and stored in $20 \mathrm{mM}$ Tris, $\mathrm{pH}$ 8.0, $100 \mathrm{mM} \mathrm{NaCl}$, and $10 \%$ glycerol. To initiate the assay, MGLL (4 $\mathrm{nM}$ ) was added to the assay system consisting of ABD-1970 (62.5-3000 $\mathrm{nM}$ ), $10 \mu \mathrm{M}$ butyl resorufin substrate (Sigma-Aldrich), $200 \mathrm{mM} \mathrm{KCl}, 1 \mathrm{mM}$ EDTA, and $50 \mathrm{mM}$ Hepes ( $\mathrm{pH}$ 7.0) in a total volume of $100 \mu$ l. Reactions without enzyme or inhibitor were included as controls. Immediately after the addition of MGLL protein, fluorescence was measured in a Neo2 multimode meter (BioTek, Winooski, VT) set for excitation at $530 \mathrm{~nm}$ and emission at $587 \mathrm{~nm}$. Data were collected at room temperature over 10 minutes, with individual reads every 12-13 seconds. The MGLL-dependent hydrolysis of the butyl resorufin substrate was monitored continuously to provide a progress curve for the rate of enzyme inactivation at varying inhibitor concentrations. The progress curve data were corrected by subtracting the average of four reactions run without enzyme. Nonlinear fitting (GraphPad Prism) of these corrected progress curves to a single exponential equation $\left[Y=Y_{0}+\left(\left(\right.\right.\right.$ plateau $\left.\left.-Y_{0}\right) \times\left(1-\exp \left(-K_{\text {obs }} \times x\right)\right)\right]$ provided the first-order rate constant, $k_{\text {obs }}$, at each concentration of inhibitor tested. The $k_{\text {obs }}$ value was plotted against ABD-1970 concentration and the curve was fit using the following equation to obtain $k_{\text {inact }}$ and $K_{\mathrm{i}}^{\text {app }}: k_{\text {obs }}=k_{\text {inact }}[I] /\left([I]+K_{\mathrm{i}}^{\text {app }}\right)$. The true $K_{\mathrm{i}}$ was subsequently calculated using the following equation: $K_{\mathrm{i}}^{\text {app }}=K_{\mathrm{i}}(1+$ $[S] / K_{\mathrm{m}}$ ) (Copeland, 2000). Each experiment was performed with quadruplicate assay points and the entire experiment was repeated three times.

In Vitro Reversibility of Inhibition and Recovery of Activity in Cultured Cells. The persistence of inhibition of human MGLL by ABD-1970 in vitro was evaluated after removal of free compound by multiple rounds of centrifugation. Human embryonic kidney 293 cells containing the SV40 T-antigen were grown in Dulbecco's modified Eagle's medium (Gibco, Grand Island, NY) containing 10\% fetal calf serum (FCS; Omega Scientific, Tarzana, CA) at $37^{\circ} \mathrm{C}$ under $5 \% \mathrm{CO}_{2}$ to approximately $60 \%$ confluence and were transfected with full-length human MGLL with an N-terminal FLAG epitope tag or an empty vector (pFLAG-CMV-6b) using the Fugene 6 reagent (Promega, Madison, WI). After 48 hours, cell membrane lysates were prepared as described in the Supplemental Methods, and an aliquot (490 $\mu \mathrm{g}$ in $490 \mu \mathrm{l}$ PBS) was treated with $1 \mu \mathrm{M} \mathrm{ABD}-1970(10 \mu \mathrm{l}$ of $50 \mu \mathrm{M})$ or DMSO for 30 minutes at $37^{\circ} \mathrm{C}$. Cell lysates were subjected to two rounds of centrifugation $\left(100,000 \mathrm{~g}\right.$ for 30 minutes at $\left.4^{\circ} \mathrm{C}\right)$ and the membrane lysates were collected immediately (time $=0$ hours), after 8 hours, and after 24 hours at room temperature and rapidly frozen on dry ice. Enzymatic activity of human MGLL was assessed in these samples after resuspension in PBS to $1 \mathrm{mg} / \mathrm{ml}$ using gel-based ABPP with the FP-Rh probe.

The recovery of MGLL activity in cultured human PC3 cells was evaluated after treatment with ABD-1970 and removal of free compound. PC3 cells were grown to $70 \%-80 \%$ confluence in $\mathrm{F}-12 \mathrm{~K}$ medium (Gibco) supplemented with $10 \% \mathrm{FCS}$ at $37^{\circ} \mathrm{C}$ with $5 \% \mathrm{CO}_{2}$, washed twice with PBS, and then incubated for 30 minutes at $37^{\circ} \mathrm{C}$ with serum-free cell culture medium containing ABD-1970 $(10 \mathrm{nM})$ or DMSO (0.01\% final concentration). Cells were washed with PBS to 
remove unbound ABD-1970, fresh media containing 10\% FCS were added, and cells were incubated at $37^{\circ} \mathrm{C}$ with $5 \% \mathrm{CO}_{2}$ for up to 72 hours. Cells were harvested at $0.5,6,24,48$, and 72 hours after the removal of ABD-1970 and cell lysates were prepared. MGLL activity in ABD-1970 - or DMSO-treated PC3 lysates was then assessed using gel-based ABPP analysis with the JW912 probe. The average recovery rate of MGLL activity was calculated by linear regression analysis using Prism software. The slope of the fit gave the average MGLL activity recovery rate in percent MGLL activity per hour.

\section{In Vivo Studies}

All animal experiments at Abide Therapeutics were performed in accordance with the guidelines outlined by the Guide for the Care and Use of Laboratory Animals and the Animal Welfare Act and were approved by the Explora Biolabs Institutional Animal Care and Use Committee. Animal experiments carried out at external institutions were performed in accordance with the individual institutions and their respective country's policies governing the ethical and humane treatment of laboratory animals. In all studies, animals were maintained under a 12-hour/12-hour light/dark cycle and allowed free access to food and water throughout the duration of the experiments.

Dose and Time-Course Effects of ABD-1970 in Rodents. Male ICR mice (Envigo, Livermore, CA) and male Sprague-Dawley rats (Charles River Laboratories, Wilmington, MA) aged 6-10 weeks at the time of dosing were administered ABD-1970 by oral gavage ( $5 \mathrm{ml} / \mathrm{kg}$ volume). ABD-1970 was prepared fresh on the day of dosing in $0.5 \%$ methylcellulose (400cP; Sigma-Aldrich) vehicle for the rat doseresponse study or in 7:2:1 polyethylene glycol 400/ethanol/PBS vehicle for the rat time-course and mouse studies. Maximal dispersal of the compound was achieved by bath and probe sonication until a fine white suspension was formed. Animals were administered single oral doses of ABD-1970 (0.1-30 mg/kg for mice and 0.1-10 mg/kg for rats). At specified time points after ABD-1970 administration, animals were anesthetized with isoflurane, and blood samples were collected by cardiac puncture into syringes precoated with EDTA $(500 \mathrm{mM}$ disodium; Teknova, Hollister, CA). After blood collection, animals were killed by decapitation, and the brains were removed and rinsed in PBS before freezing in liquid nitrogen. One brain hemisphere was submitted for ABD-1970 concentration analysis by LC-MS/MS, and the other hemisphere was further dissected to remove two adjacent forebrain sections (approximately 100-200 mg) and was submitted for analysis of MGLL inhibition by ABPP with the FP-Rh probe, as described above, and for brain lipid concentrations by LC-MS/MS, as described below.

Because of the instability of ABD-1970 in rodent blood and plasma that is thought to be a consequence of carboxylesterase (CES) enzymes, which are known to metabolize ester and carbamate xenobiotics and are more abundant in rodent plasma than other higher mammal species, including dogs, primates, and humans (Berry et al., 2009), blood samples were immediately placed into an equal volume of acetonitrile to stabilize ABD-1970. After mixing, the samples were centrifuged $(17,000 \mathrm{~g}$ for 2 minutes), and the resulting whole blood supernatants were transferred and submitted to LC-MS/MS analysis for whole blood ABD-1970 measurements.

Brain Lipid Analysis. Quantitation of endocannabinoid and eicosanoid lipids in brain tissue after organic extraction was performed by LC-MS/MS analysis on an Agilent $1260 \mathrm{LC}$ system coupled to an Agilent 6460 Triple Quadrupole mass spectrometer (Agilent Technologies, Santa Clara, CA). Full experimental details are presented in the Supplemental Methods.

Formalin Pain Model. Male Sprague-Dawley rats (171-235 g at the time of dosing; Harlan, Huntingdon, UK) were administered an intraplantar injection of formalin $(50 \mu \mathrm{l}, 2.5 \%)$ into the dorsal surface of the right hindpaw to induce acute pain (Saretius Ltd., Reading, UK). Immediately after, paw licking behavior, a correlate of spontaneous pain (Abbott et al., 1995), was tracked using the Laboras (Laboratorium Animal Behavior Observation, Registration and
Analysis System; Metris B.V., Hoofddorp, The Netherlands) automated rodent behavioral tracking system, which was validated to differentiate and automatically recognize behaviors and locomotor activity of individually housed rats. Animals injected with formalin were immediately placed into the Laboras cages $[22.5 \mathrm{~cm}$ (width) $\times$ $42.5 \mathrm{~cm}$ (length) $\times 19.0 \mathrm{~cm}$ (height)] and data on the duration of paw licking behavior (in seconds) were collected from two distinctive phases: 1 ) the early nociceptive phase occurring $0-5$ minutes after formalin injection and 2) the late phase thought to be associated with central sensitization, which peaks 10-30 minutes after formalin injection.

To establish a pharmacokinetic (PK)/pharmacodynamic (PD) relationship, animals were administered vehicle ( $0.5 \%$ methylcellulose), ABD-1970 (1, 3, and $10 \mathrm{mg} / \mathrm{kg}$ ), or the positive control pregabalin $(60 \mathrm{mg} / \mathrm{kg}) 240$ minutes before formalin injection. Forty-five minutes after formalin injection and behavioral monitoring and approximately 285 minutes after treatment, animals were anesthetized with isoflurane and blood samples were collected by cardiac puncture. After blood collection, animals were killed by decapitation and the brains were removed. Samples were dissected and processed as described above for analysis of MGLL inhibition by ABPP and ABD-1970 and 2-AG concentrations by LC-MS/MS.

For the ABD-1970 and pregabalin combination study, male Sprague-Dawley rats (214-235 g; Harlan) were administered ABD$1970(2 \mathrm{mg} / \mathrm{kg})$ by oral gavage $(5 \mathrm{ml} / \mathrm{kg}$ volume $)$ immediately followed by a separate oral administration ( $5 \mathrm{ml} / \mathrm{kg}$ volume) of pregabalin $(10 \mathrm{mg} / \mathrm{kg}) 240$ minutes before formalin administration. For the monotherapy control groups, ABD-1970 or pregabalin was administered along with separate oral gavage administrations of vehicle $(0.5 \%$ methylcellulose). Paw licking behavior was monitored for 30 minutes using the Laboras behavior tracking system. For the ABD-1970 and morphine combination study, male Sprague-Dawley rats (225-269 g at the time of dosing; Harlan) were administered vehicle $(0.5 \%$ methylcellulose) or ABD-1970 (1 mg/kg) by oral gavage. After 210 minutes, vehicle (saline) or morphine $(2.49 \mathrm{mg} / \mathrm{kg})$ was administered by subcutaneous injection. After an additional 30 minutes (240 minutes after ABD-1970 and 30 minutes after morphine), formalin was administered into the hindpaw and paw licking behavior was monitored using the Laboras behavior tracking system.

CFA Model of Inflammatory Pain. Male Sprague-Dawley rats (200-230 g; Charles River Laboratories, Beijing, China) were administered an intraplantar injection of CFA $(50 \mu \mathrm{l}, 4 \mathrm{mg} / \mathrm{ml})$ into the dorsal surface of the left hindpaw to induce inflammation and pain (Pharmaron, Beijing, China). Twenty-four hours after CFA injection, animals were administered vehicle (0.5\% methylcellulose) or ABD$1970(1,3$, and $10 \mathrm{mg} / \mathrm{kg})$ by oral gavage.

Mechanical allodynia in the CFA-injected paw was measured prior to CFA injection and 0 (24 hours after CFA and before vehicle or ABD1970), 2, 4, and 6 hours after compound administration by determining withdrawal thresholds to an electronic von Frey instrument (Bioseb, Vitrolles, France) applied perpendicularly with increasing force to the plantar surface of the paw. The threshold for paw withdrawal was calculated by taking the average of two to three repeated von Frey applications. Observers were blinded to the identity of the treatment groups.

Plantar Incision Model of Postoperative Pain. Postoperative pain was induced in male Sprague-Dawley rats (220-250 g; Charles River Laboratories) as described previously (Brennan et al., 1996) (Pharmaron). Briefly, rats were anesthetized with isoflurane and placed in a dorsal recumbent position, and the plantar surface of the left hind foot was draped and prepared aseptically. On the plantar surface of the foot, a $1-\mathrm{cm}$ incision was made at $0.5 \mathrm{~cm}$ from the edge of the heel and extending distally through the skin and fascia. The plantaris muscle was exposed by blunt dissection, elevated and incised longitudinally, and the skin was closed with two interrupted mattress sutures (3-0, polydioxanone).

Animals were administered vehicle ( $0.5 \%$ methylcellulose) or ABD$1970(20 \mathrm{mg} / \mathrm{kg})$ by oral gavage 60 minutes before anesthesia induction and plantar incision. Buprenorphine $(0.03 \mathrm{mg} / \mathrm{kg})$ was administered 
by intraperitoneal injection immediately before the procedure. Mechanical allodynia in the operated paw was measured before compound administration $(t=0)$ to establish presurgical baseline withdrawal thresholds, and at 1, 3, and 5 hours after incision using an electronic von Frey instrument (Bioseb). Observers were blinded to the identity of the treatment groups.

Chronic Constriction Injury Model of Peripheral Neuropathic Pain. Chronic constriction injury (CCI) on the left common sciatic nerve was performed in male Sprague-Dawley rats (Harlan) to induce neuropathic pain as described previously (Bennett and Xie, 1988) (Aquila BioMedical Ltd., Edinburgh, UK). After recovery from anesthesia, animals were recovered for 7 days before behavioral testing was initiated.

Mechanical allodynia was assessed in rats by measuring the withdrawal threshold to von Frey filaments of increasing diameter and force. Animals were placed in a cage with a wire mesh bottom, and a series of calibrated von Frey filaments [3.84 (0.6 g), $4.17(1.4 \mathrm{~g}), 4.56$ $(3.8 \mathrm{~g}), 4.93(7.1 \mathrm{~g}), 5.18(12.4 \mathrm{~g})$, and $5.46(20.5 \mathrm{~g})]$ was applied to the plantar surface of the left hind paw. Each paw was tested six times with von Frey fibers presented consecutively. A minimum of three of six withdrawals was considered a positive response. The middle filament (4.56) was applied first; if a positive response was observed, the next weaker filament was then tested. Conversely, if the rat did not withdraw its paw at least three times, a larger filament would be tested until a 50\% (three of six) withdrawal response was observed. Testing continued in this manner until a withdrawal threshold was reached.

von Frey thresholds were measured prior to CCI on all rats to establish a baseline withdrawal threshold and then after the CCI procedure on days 7 and 14 to monitor the development of mechanical allodynia. Animals with left paw withdrawal thresholds to von Frey fibers $\leq 8 \mathrm{~g}$ were deemed to have developed mechanical allodynia and were included in the study. Allocation to treatment groups was based on each animal's pretreatment withdrawal threshold measured on day 14 .

On day 14 or 15 , animals received a single oral gavage dose of vehicle (0.5\% methylcellulose), ABD-1970, or pregabalin $(10 \mathrm{ml} / \mathrm{kg}$ volume); 240 minutes later, mechanical allodynia was measured to determine treatment effects. All observers were blinded to the identity of the treatment groups.

Serotonin-Induced Scratching Model of Pruritus. The day before the experiment, female Sprague-Dawley rats (202-278 g; Janvier Laboratories, Le Genest-Saint-Isle, France) were shaved on the rostral part of the back and then placed individually into clear cylindrical observation chambers (height $=35 \mathrm{~cm}$, diameter $=19 \mathrm{~cm}$ ) for at least 60 minutes to habituate them to the testing environment (Porsolt, Le Genest-Saint-Isle, France). On the day of the experiment, scratching was induced by intradermal injection of serotonin $(50 \mu \mathrm{l}$, $50 \mu \mathrm{g} / \mathrm{rat}$ ) into the rostral part of the rat's back.

To test the effects of ABD-1970 on serotonin-induced scratching, animals were administered vehicle (0.5\% methylcellulose) or ABD$1970(1,2$, or $10 \mathrm{mg} / \mathrm{kg}$ ) by oral gavage ( $5 \mathrm{ml} / \mathrm{kg}$ volume) 240 minutes before serotonin injection. The positive control naltrexone $(1 \mathrm{mg} / \mathrm{kg})$ or its respective vehicle $(0.9 \%$ saline $)$ was administered subcutaneously 15 minutes before serotonin. Scratching bouts were monitored for 30 minutes after serotonin injection by an observer blinded to the experimental groups. A single scratching bout was defined as one or more rapid movements of the hindpaws to the area around the site of serotonin injection ending with licking or biting of the toes and/or placement of the hindpaw on the floor of the observation chamber.

Locomotor Activity. Open field behavior was monitored in male Sprague-Dawley rats 240 minutes after oral administration of ABD1970 ( 1 and $10 \mathrm{mg} / \mathrm{kg}, 0.5 \%$ methylcellulose vehicle) or 15 minutes after subcutaneous administration of the CB agonist WIN 55,212-2 (3 mg/kg, 1\% Tween 80/99\% saline vehicle) at Saretius Ltd. To control for the different administration times and routes, all animals received counterbalanced vehicle administrations 240 minutes $(0.5 \%$ methylcellulose, p.o.) or 15 minutes ( $1 \%$ Tween $80 / 99 \%$ saline, s.c.) prior to behavior assessment. Locomotor activity, rearing, and grooming were monitored for 40 minutes using the Laboras automated behavioral tracking system.

\section{Prostanoid Production in Stimulated Human Blood and Cells}

Lipopolysaccharide-Stimulated Whole Blood. Normal human blood was obtained from The Scripps Research Institute Normal Blood Donor Service (La Jolla, CA) and was approved by The Scripps Research Institute institutional review board. Blood samples from two donors per experiment (one male donor and one female donor) were prepared by diluting equal parts heparinized whole blood and serumfree RPMI medium (v/v). Diluted samples $(4 \mathrm{ml})$ were treated with ABD-1970 $(0.03-30 \mu \mathrm{M})$, indomethacin $(10 \mu \mathrm{M})$, rofecoxib $(10 \mu \mathrm{M})$, or vehicle (DMSO) for 30 minutes at $37^{\circ} \mathrm{C}$ in 24 -well plates and were then stimulated with $30 \mathrm{ng} / \mathrm{ml}$ lipopolysaccharide (LPS; $E$. coli serotype 0111:B4, no. 581-012-L002; Enzo Life Sciences, New York, NY) or vehicle for approximately 24 hours at $37^{\circ} \mathrm{C}$. To collect plasma, well contents were transferred to a $15-\mathrm{ml}$ conical tube and centrifuged at $1500 \mathrm{rpm}$ for 5 minutes to pellet erythrocytes. The clear supernatant was isolated, transferred to a glass vial, and frozen at $-80^{\circ} \mathrm{C}$ prior to LC-MS/MS analysis as described in the Supplemental Methods.

Ionomycin-Stimulated Whole Blood. Blood from one female donor was prepared by diluting equal parts heparinized whole blood and serum-free RPMI (v/v). Diluted samples $(1 \mathrm{ml})$ were treated with ABD-1970 $(0.3$ and $3 \mu \mathrm{M})$, indomethacin $(10 \mu \mathrm{M})$, rofecoxib $(10 \mu \mathrm{M})$, or vehicle (DMSO) for 30 minutes at $37^{\circ} \mathrm{C}$ in 24 -well plates and were then stimulated with $30 \mu \mathrm{M}$ of the calcium ionophore ionomycin (Calbiochem, San Diego, CA) or vehicle for 30 minutes at $37^{\circ} \mathrm{C}$. To collect plasma, plates were centrifuged at $1500 \mathrm{rpm}$ for 5 minutes to pellet erythrocytes. The clear supernatant was isolated, transferred to a glass vial, and frozen at $-80^{\circ} \mathrm{C}$ prior to LC-MS/MS analysis as described in the Supplemental Methods.

Interleukin-1 $\beta$-Stimulated Human Umbilical Vein Endothelial Cells. Primary human umbilical vein endothelial cells (HUVECs) pooled from multiple donors were obtained from Gibco (no. C-015-10c, lot 1150190, mycoplasma negative) and expanded in Medium 200 (no. R-001-100; Gibco) containing low serum growth supplement (no. S-003-10; Gibco). Cells were plated in six-well culture dishes $\left(1 \times 10^{6}\right.$ million cells per well) until well attached ( 6 hours), washed twice with phenol- and serum-free Medium 200PRF (no. $\mathrm{M}-200 \mathrm{PRF}-500$; Gibco), and then pretreated for 30 minutes at $37^{\circ} \mathrm{C}$ in 95\% $\mathrm{O}_{2}$ and $5 \% \mathrm{CO}_{2}$ in Medium 200PRF containing one of the following: ABD-1970 $(0.1,10$, or $100 \mathrm{nM})$, indomethacin $(500 \mathrm{nM})$, rofecoxib (500 nM), or DMSO vehicle. Cells were stimulated with interleukin (IL)- $1 \beta$ ( $1 \mathrm{ng} / \mathrm{ml}$, no. 201-LB-005; R\&D Systems, Minneapolis, MN) for approximately 18 hours at $37^{\circ} \mathrm{C}$ in $95 \% \mathrm{O}_{2}$ and $5 \% \mathrm{CO}_{2}$. Media were collected, centrifuged to remove nonadherent cells, and frozen at $-80^{\circ} \mathrm{C}$ prior to LC-MS/MS analysis as described in the Supplemental Methods. HUVECs were collected by scraping in cold $\mathrm{PBS}$ and frozen at $-80^{\circ} \mathrm{C}$ for subsequent $\mathrm{ABPP}$ analysis with $\mathrm{FP}-\mathrm{Rh}$ to confirm MGLL target engagement and inhibitor selectivity.

Platelet Aggregation. The effects of ABD-1970 on platelet aggregation induced by collagen were evaluated essentially as previously described (Born, 1962) (Eurofins Panlabs, Taipei City, Taiwan). Human platelet-rich plasma $\left(60.0 \pm 10 \mathrm{~kg} ; 6\right.$ to $7 \times 10^{8}$ platelets $/ \mathrm{ml}$ ) was treated with $0.3,3$, or $30 \mu \mathrm{M}$ ABD-1970 or $3 \mu \mathrm{M}$ indomethacin for 5 minutes at $37^{\circ} \mathrm{C}$. Collagen $(10 \mu \mathrm{g} / \mathrm{ml})$ was added to induce aggregation, which was measured by an optical aggregometer.

\section{Statistical Analyses}

Results are presented as the mean \pm S.E.M. Treatment effects on formalin-induced paw licking were analyzed by one-way analysis of variance followed by the Dunnett post-test or Newman-Keuls multiple-comparison tests, as warranted. The CCI study in which the effect of treatment on von Frey thresholds versus pretreatment 
thresholds was compared was analyzed by a paired Wilcoxon test. Treatment effects on all additional data sets were analyzed by analysis of variance and the Dunnett post-test. Differences were considered statistically significant at $P<0.05$. All statistical analyses were carried out using Prism (version 6), SPSS (version 17.0; IBM, Armonk, NY), or Statistica (version 10.1; Statsoft, Palo Alto, CA) software.

\section{Results}

\section{ABD-1970 Is a Potent and Selective Inhibitor of MGLL across Species}

ABD-1970 (Fig. 1) is a member of the carbamate class of MGLL inhibitors, which have been shown to act via covalent carbamylation of the active-site serine residue (Long et al., 2009a; Chang et al., 2012; Niphakis et al., 2012; Griebel et al., 2015; Butler et al., 2017). Characterization of ABD-1970 potency and selectivity leveraged ABPP, a chemical proteomics platform that utilizes active site-directed chemical probes to evaluate the activity of entire enzyme families in parallel in native biologic matrices (Simon and Cravatt, 2010). In vitro treatment of tissue or cell homogenates with $\mathrm{ABD}$ 1970, followed by incubation with ABPP probes, SDS-PAGE analysis, and in-gel fluorescence imaging, allows for the direct visualization of the targets of ABD-1970. In this gel-based $\mathrm{ABPP}$ platform, quantification of serine hydrolase activity is based on the intensity of probe labeling, and inhibition is measured as a reduction of probe labeling compared with control samples.

ABD-1970 (0.001-10 $\mu \mathrm{M}, 30$ minutes, $\left.37^{\circ} \mathrm{C}\right)$ was analyzed by $\mathrm{ABPP}$ in brain tissue homogenates from mice, rats, rabbits, dogs, monkeys, and humans, as well as in lysates prepared from PC3 cells. Brain tissue was chosen for profiling because it represents an important therapeutic target organ and exhibits high activity of many serine hydrolases, including MGLL. PC3 cells are a rich source of human MGLL, as well as of ABHD6, an off-target common to published MGLL inhibitors (Long et al., 2009a; Chang et al., 2012; Niphakis et al., 2012; Griebel et al., 2015; Butler et al., 2017). Inhibition of MGLL activity was observed after pretreatment with ABD-1970 in brain tissue homogenates of all species and PC3 cell lysates, with average $\mathrm{IC}_{50}$ values of $<20 \mathrm{nM}$ (average values presented in Table 1 and representative ABPP gel images and MGLL activity quantitation in Fig. 2). MGLL migrates as multiple bands in the ABPP gels, and each band was equally sensitive to ABD-1970. ABD-1970 showed excellent selectivity in all species tested, crossreacting with only a single off-target

\section{TABLE 1}

In vitro potency of ABD-1970 against MGLL across species

ABD-1970 $(0-10 \mu \mathrm{M})$ was preincubated with the source material listed for 30 minutes at $37^{\circ} \mathrm{C}$ prior to ABPP analysis. Data represent the mean \pm S.E.M. from several independent experiments.

\begin{tabular}{llcc}
\hline \multicolumn{1}{c}{ Species } & \multicolumn{1}{c}{ Source } & $n$ & MGLL IC $_{50}$ \\
\hline & & \multicolumn{2}{c}{$\mu M$} \\
Mouse & Brain & 4 & $0.015 \pm 0.003$ \\
Rat & Brain cortex & 3 & $0.003 \pm 0.001$ \\
Rabbit & Brain & 3 & $0.003 \pm 0.001$ \\
Dog & Brain cortex & 3 & $0.007 \pm 0.001$ \\
Monkey & Brain cortex & 3 & $0.018 \pm 0.003$ \\
Human & Brain prefrontal cortex & 3 & $0.013 \pm 0.003$ \\
& PC3 cell lysate & 4 & $0.018 \pm 0.003$ \\
\hline
\end{tabular}

(ABHD6) in mouse, rat, rabbit, dog, and monkey brain homogenates, with $\mathrm{IC}_{50}$ values of $3.2,0.8,0.6,1.4$, and $3 \mu \mathrm{M}$, respectively. ABHD6 was inhibited in human PC3 cells with an $\mathrm{IC}_{50}$ of $2.5 \mu \mathrm{M}$, whereas ABHD6 activity was not detected by gel-based ABPP in human brain tissue. Notably, at the concentrations tested, ABD-1970 did not inhibit fatty acid amide hydrolase. Since the carbamate functional group of ABD-1970 could be subject to hydrolysis, we evaluated the activity of the amine analog ABD-0038 (Supplemental Fig. 1A) using ABPP. Demonstrating that the hexafluoroisopropanol carbamate functional group is required for the activity of ABD1970, ABD-0038 did not inhibit MGLL or other serine hydrolases in mouse, rat, and human brain tissue (Supplemental Fig. 1, B-D).

The cellular activity of ABD-1970 was evaluated in two human systems: cultured PC3 cells and PBMCs isolated from human whole blood. When added to the cell culture media, ABD-1970 (0.001-10 $\mu \mathrm{M}, 30$ minutes, $\left.37^{\circ} \mathrm{C}\right)$ was a potent inhibitor of MGLL in intact PC3 cells, displaying an $\mathrm{IC}_{50}$ value of $3.2 \mathrm{nM}$. MGLL was also inhibited in human PBMCs after treatment of whole blood with ABD-1970 (0.03-30 $\mu \mathrm{M}$, 30 minutes, $37^{\circ} \mathrm{C}$ ). In this human blood assay, which provides a basis for a clinical peripheral biomarker assay, ABD-1970 treatment inhibited MGLL with an average $\mathrm{IC}_{50}$ value of $62 \mathrm{nM}$ (Supplemental Fig. 2A).

To amplify endocannabinoid signaling and modulate neurotransmission clinically, an MGLL inhibitor must effectively prevent the breakdown of 2-AG in the human nervous system. MGLL was previously shown to be the major 2-AG hydrolase in the rodent brain both in vitro and in vivo (Blankman et al., 2007; Long et al., 2009a). Here, we used ABD-1970 to evaluate the contribution of MGLL to 2-AG catabolism in human brain tissue. Inhibition of 2-AG hydrolysis by ABD-1970 (0.0003-1 $\mu \mathrm{M}, 30$ minutes, $37^{\circ} \mathrm{C}$ ) was assessed using an MS-based 2-AG substrate assay, which revealed that approximately $95 \%$ of the 2-AG hydrolytic activity in the particulate fraction of the human brain cortex was blocked by ABD-1970 with an $\mathrm{IC}_{50}$ of 3 nM (Supplemental Fig. 2B).

Some carbamate/urea inhibitors of serine hydrolases can display slow reversibility, presumably due to hydrolytic turnover of the carbamylated active-site serine residue (Bar-On et al., 2002; Keith et al., 2015). We therefore evaluated the persistence of the ABD-1970-MGLL interaction in vitro by assessing enzyme activity over a 24-hour period after removal of excess compound. After treatment with ABD1970 ( $1 \mu \mathrm{M}, 30$ minutes, $37^{\circ} \mathrm{C}$ ), human MGLL in transfected lysates of human embryonic kidney 293 cells containing the SV40 T-antigen remained inhibited by greater than $95 \%$ for up to 24 hours after removal of free compound (Supplemental Fig. 2C). Based on these measurements, we conclude that the presumed carbamylated adduct formed between human MGLL and ABD-1970 is highly stable over a 24-hour time period, with very little hydrolysis and reactivation of the enzyme.

Considering that ABD-1970 is an irreversible inhibitor of MGLL, we determined a second-order rate constant (or $k_{\text {inact }}$ / $K_{\mathrm{i}}$ ) value for the compound, which provides a measurement of potency that is independent of substrate concentration and preincubation time. Using a fluorescent substrate assay, ABD-1970 displayed time-dependent inhibition of purified recombinant human MGLL (Supplemental Fig. 2D). The progress curves were fit to a first-order exponential to 
A

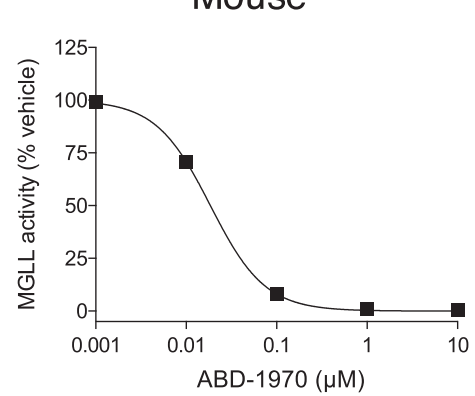

D

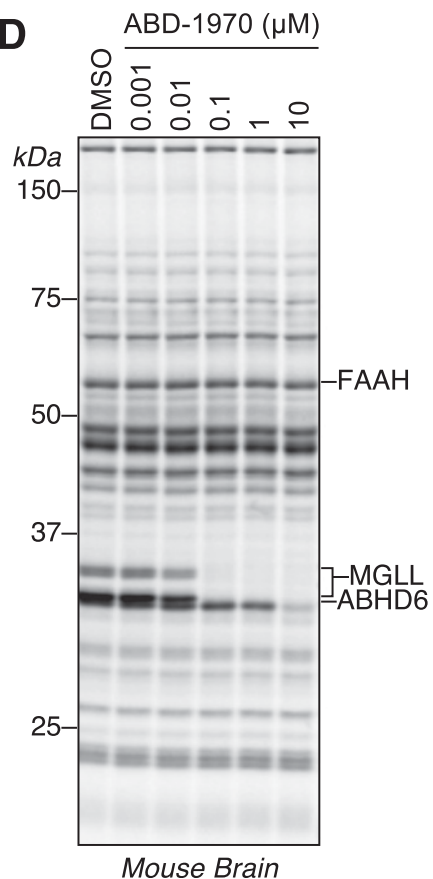

B

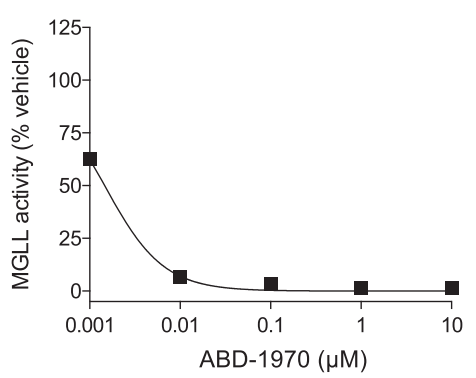

E

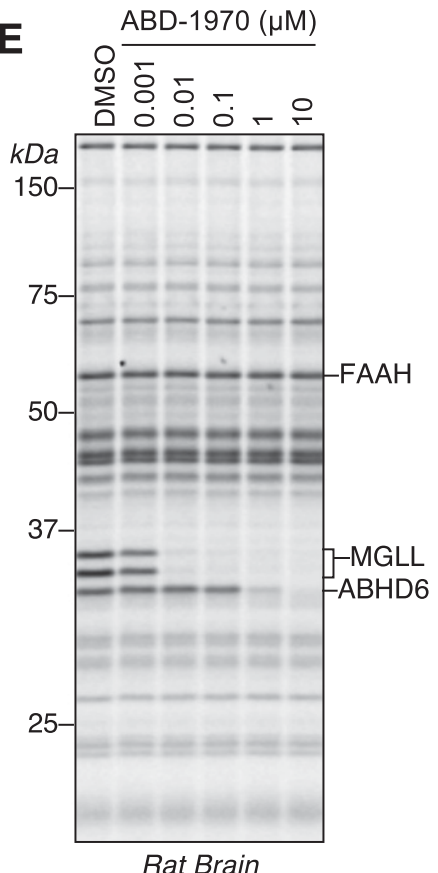

C

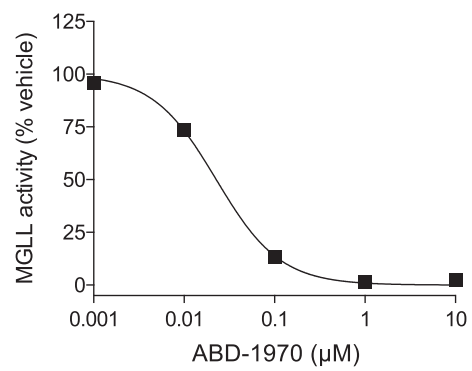

F

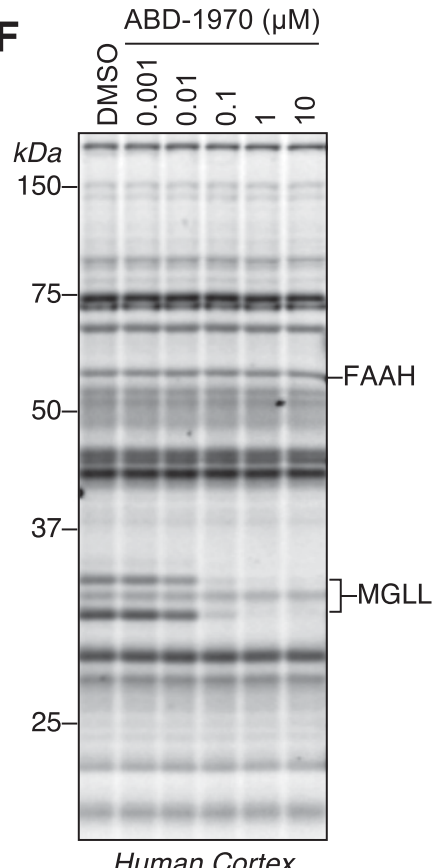

Fig. 2. Potent and selective inhibition of MGLL in brain tissue by ABD-1970. (A-F) MGLL activity quantitation (A-C) and representative ABPP gels (D-F) in mouse, rat, and human brain membrane homogenates treated with ABD-1970 (30 minutes, $\left.37^{\circ} \mathrm{C}\right)$. Mean $\mathrm{IC}_{50}$ values are reported in Table 1. ABHD6, $\alpha / \beta$ hydrolase domain containing 6 FAAH, fatty acid amide hydrolase MGLL, monoacylglycerol lipase.

determine the observed first-order rate constant for enzyme inactivation $\left(k_{\mathrm{obs}}\right)$ at each concentration of ABD-1970 (Supplemental Fig. 2E). Nonlinear curve fitting of the $k_{\text {obs }}$ versus ABD-1970 concentration plot allowed determination of $k_{\text {inact }}$, $K_{\mathrm{i}}$, and the second-order rate constant $k_{\text {inact }} / K_{\mathrm{i}}$. ABD-1970 displayed an average $k_{\text {inact }}$ value of $0.006 \mathrm{~s}^{-1}$, an average $K_{\mathrm{i}}$ value of $0.3 \mu \mathrm{M}$, and an average $k_{\text {inact }} / K_{\mathrm{i}}$ value of approximately $19,000 \mathrm{M}^{-1} \mathrm{~s}^{-1}$ against human MGLL.

For irreversible enzyme inhibitors, the recovery of enzymatic activity in vivo and in intact cells is largely mediated by protein biosynthesis. To approximate the rate of recovery of MGLL activity after irreversible inhibition in human cells, we measured MGLL activity in cultured PC3 cells for up to 72 hours after incubation with ABD-1970 (10 nM, 30 minutes, $37^{\circ} \mathrm{C}$ ) and removal of excess compound. ABD-1970 treatment inhibited MGLL activity by approximately $90 \%$ at 30 minutes post-treatment, and near-complete recovery of MGLL activity was observed 72 hours after free ABD-1970 was removed from the culture media (Supplemental Fig. 2F). The average recovery rate for MGLL activity over the course of the experiment (0.5-72 hours) was approximately $1 \%$ activity per hour.

\section{ABD-1970 Is Highly Selective for MGLL in Human Tissues and Cells}

A comprehensive characterization of the selectivity of $\mathrm{ABD}$ 1970 across the serine hydrolase class was performed in human tissues and cells using both gel-based and MS-based ABPP methods. In vitro treatment of human brain (prefrontal cortex), liver, kidney, skin, and lung homogenates or PBMC lysates with ABD-1970 (10 $\mu \mathrm{M}, 30$ minutes, $\left.37^{\circ} \mathrm{C}\right)$ revealed selective inhibition of MGLL by gel-based ABPP with the FP-Rh probe (Fig. 3A). The only off-target of ABD-1970 observed in these tissues was the xenobiotic metabolizing enzyme CES1.

The same principles of ABPP can be applied to high-content MS-based proteomic platforms for identification and quantification of protein targets of inhibitors. Here, human brain homogenates were treated with $\mathrm{ABD}-1970(0.01-10 \mu \mathrm{M})$ or DMSO vehicle for 30 minutes at $37^{\circ} \mathrm{C}$ and were subjected to MS-based ABPP using the FP-biotin probe to enrich active but not inhibited serine hydrolases via streptavidin-affinity chromatography (Jessani et al., 2005). Isotopic labels were incorporated into peptides from DMSO- and inhibitor-treated samples, permitting accurate quantitation of relative enzyme activities in a single MS experiment (Boersema et al., 2009). 
A

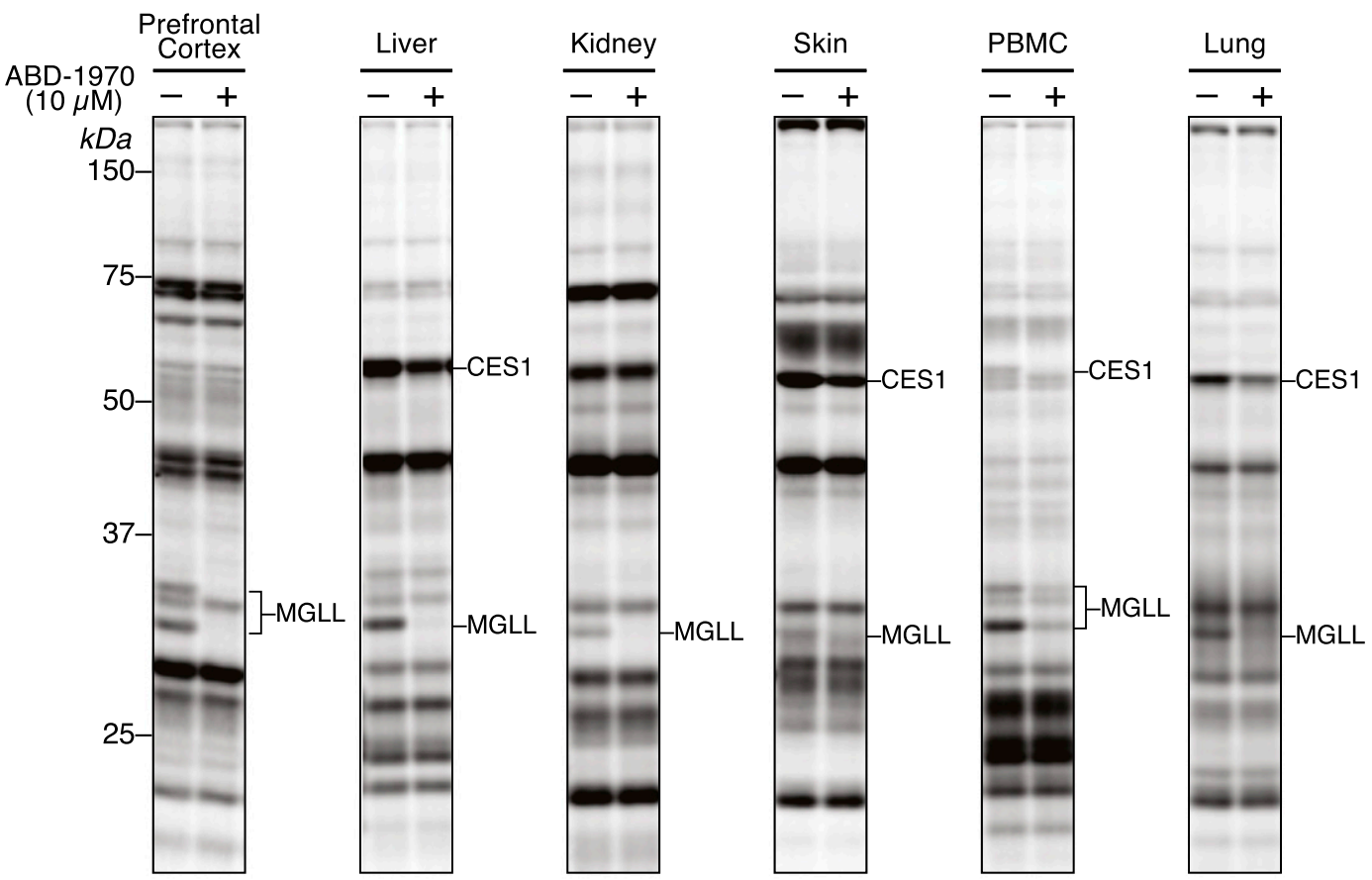

$\mathbf{B}$

Serine Hydrolases (Prefrontal Cortex)

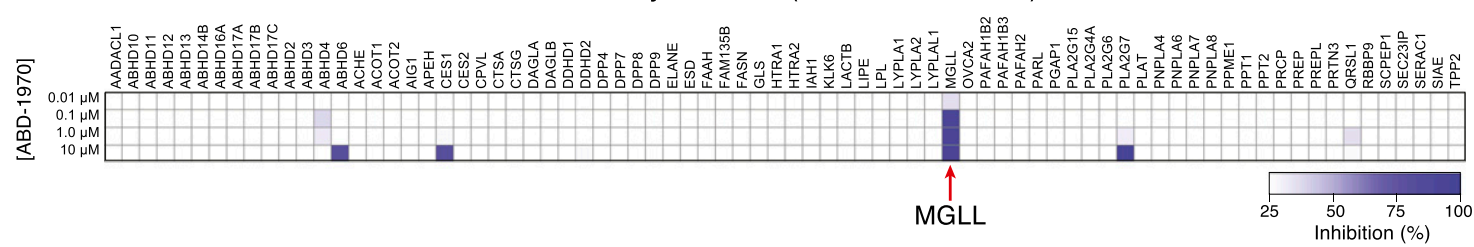

Fig. 3. ABD-1970 is highly selective for MGLL in human tissues and cells. (A and B) Selectivity of ABD-1970 ( 30 minutes at $37^{\circ} \mathrm{C}$ ) was evaluated in human tissue and cell homogenates (total homogenates containing soluble and membrane fractions) by gel-based ABPP (A) and in membrane homogenates of human prefrontal cortex by MS-based ABPP (B). AADACL1, Arylacetamide deacetylase-like $1 ; \mathrm{ABHD} 10, \alpha / \beta$ hydrolase domain-containing $10 ; \mathrm{ABHD} 11, \alpha / \beta$ hydrolase domain containing 11; ABHD12, $\alpha / \beta$ hydrolase domain containing 12; ABHD13, $\alpha / \beta$ hydrolase domain containing 13; ABHD14B, $\alpha / \beta$ hydrolase domain containing 14B; ABHD16A, $\alpha / \beta$ hydrolase domain containing $16 \mathrm{~A} ; \mathrm{ABHD} 17 \mathrm{~A}, \alpha / \beta$ hydrolase domain containing $17 \mathrm{~A} ; \mathrm{ABHD} 17 \mathrm{~B}, \alpha / \beta$ hydrolase domain containing 17B; ABHD17C, $\alpha / \beta$ hydrolase domain containing 17C; ABHD2, $\alpha / \beta$ hydrolase domain containing 2 ; ABHD3, $\alpha / \beta$ hydrolase domain containing 3; ABHD4, $\alpha / \beta$ hydrolase domain containing 4; ABHD6, $\alpha / \beta$ hydrolase domain containing 6; ACHE, Acetylcholinesterase; ACOT1, Acyl-coenzyme A thioesterase 1; ACOT2, Acyl-coenzyme A thioesterase 2; AIG1, Androgen-induced gene 1 protein; APEH, Acylamino-acid-releasing enzyme; CES1, Carboxylesterase 1; CES2, Carboxylesterase 2; CPVL, Probable serine carboxypeptidase CPVL; CTSA, Lysosomal protective protein; CTSG, Cathepsin G; DAGLA, Sn1-specific diacylglycerol lipase alpha; DAGLB, Sn1-specific diacylglycerol lipase beta; DDHD1, Phospholipase DDHD1; DDHD2, Phospholipase DDHD2; DPP4, Dipeptidyl peptidase 4; DPP7, Dipeptidyl peptidase 2; DPP8, Dipeptidyl peptidase 8; DPP9, Dipeptidyl peptidase 9; ELANE, Neutrophil elastase; ESD, S-formylglutathione hydrolase; FAAH, Fatty-acid amide hydrolase 1; FAM135B, Protein FAM135B; FASN, Fatty acid synthase; GLS, Glutaminase kidney isoform, mitochondrial; HTRA1, Serine protease HTRA1; HTRA2, Serine protease HTRA2, mitochondrial; IAH1, Isoamyl acetatehydrolyzing esterase 1 homolog; KLK6, Kallikrein-6; LACTB, Serine beta-lactamase-like protein LACTB, mitochondrial; LIPE, Hormone-sensitive lipase; LPL, Lipoprotein lipase; LYPLA1, Acyl-protein thioesterase 1; LYPLA2, Acyl-protein thioesterase 2; LYPLAL1, Lysophospholipase-like protein 1; MGLL, Monoglyceride lipase; OVCA2, Esterase OVCA2; PAFAH1B2, Platelet-activating factor acetylhydrolase IB subunit beta; PAFAH1B3, Platelet-activating factor acetylhydrolase IB subunit gamma; PAFAH2, Platelet-activating factor acetylhydrolase 2, cytoplasmic; PARL, Presenilins-associated rhomboid-like protein, mitochondrial; PGAP1, GPI inositol-deacylase; PLA2G15, Group XV phospholipase A2; PLA2G4A, Cytosolic phospholipase A2; PLA2G6, 85/88 kDa calcium-independent phospholipase A2; PLA2G7, Phospholipase A2 group VII; PLAT, Tissue-type plasminogen activator; PNPLA4, Patatin-like phospholipase domain-containing protein 4; PNPLA6, Neuropathy target esterase; PNPLA7, Patatin-like phospholipase domain-containing protein 7; PNPLA8, Calcium-independent phospholipase A2-gamma; PPME1, Protein phosphatase methylesterase 1; PPT1, Palmitoyl-protein thioesterase 1; PPT2, Lysosomal thioesterase PPT2; PRCP, Lysosomal Pro-X carboxypeptidase; PREP, Prolyl endopeptidase; PREPL, Prolyl endopeptidase-like; PRTN3, Myeloblastin; QRSL1, Glutamyl-tRNA; RBBP9, Putative hydrolase RBBP9; SCPEP1, Retinoid-inducible serine carboxypeptidase; SEC23IP, SEC23interacting protein; SERAC1, Protein SERAC1; SIAE, Sialate O-acetylesterase; TPP2, Tripeptidyl-peptidase 2

This analysis resulted in the identification and quantification of 78 cerebral human serine hydrolases. Consistent with the gel-based analyses, MGLL was completely inhibited by ABD-1970 at concentrations as low as $0.1 \mu \mathrm{M}$, and no offtargets were observed until $10 \mu \mathrm{M}$ ABD-1970, at which concentration, ABHD6, CES1, and phospholipase A2 group VII were also inhibited (Fig. 3B). ABD-1970 displays a mean $\mathrm{IC}_{50}$ of $0.013 \mu \mathrm{M}$ toward MGLL in human brain homogenates (Table 1) and therefore exhibits at least 77-fold selectivity for
MGLL against ABHD6, CES1, and phospholipase A2 group VII and at least 770-fold selectivity for MGLL versus the other 74 enzymes profiled.

\section{ABD-1970 Selectively Inhibits MGLL In Vivo and Modulates Brain Levels of 2-AG, AA, and Prostanoids}

The dose- and time-dependent effects of oral ABD-1970 administration were evaluated in both mice and rats. Single oral gavage doses of ABD-1970 $(0.1-10 \mathrm{mg} / \mathrm{kg})$ to rats 
A

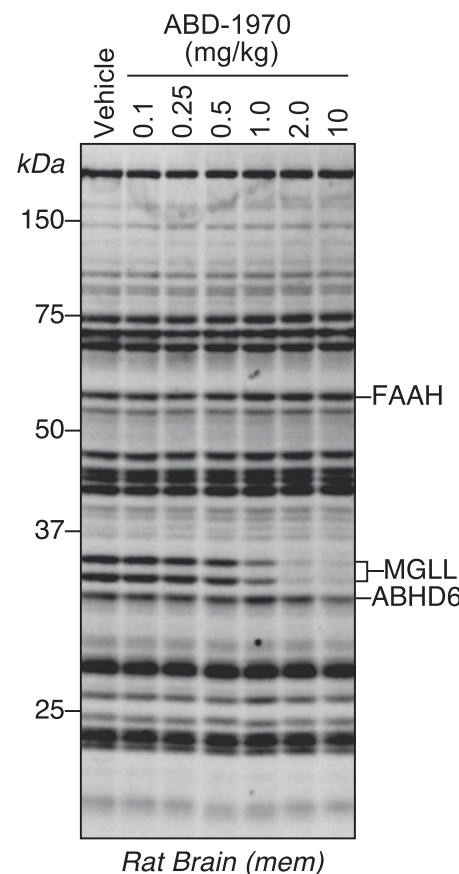

F

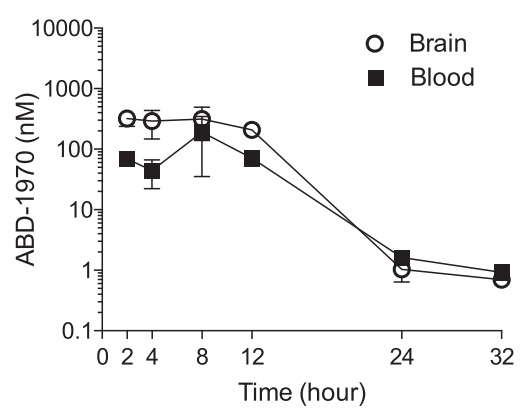

B

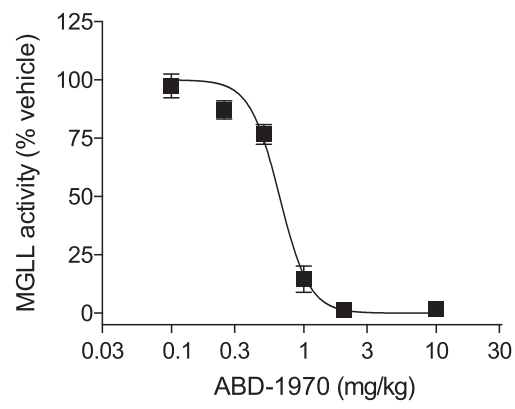

D

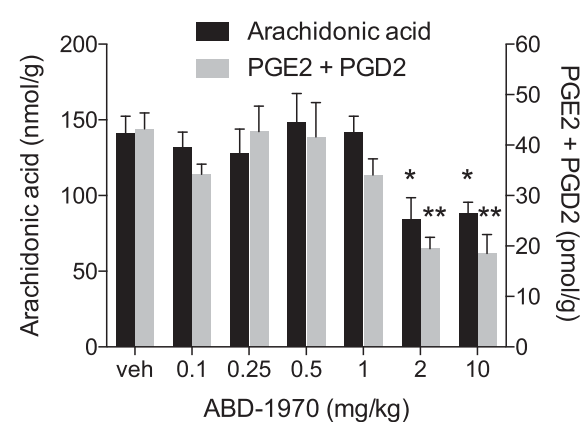

G

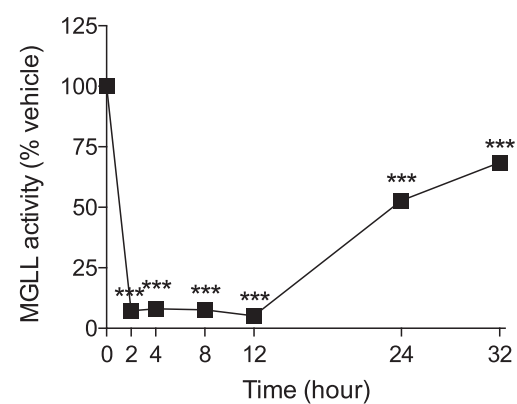

C

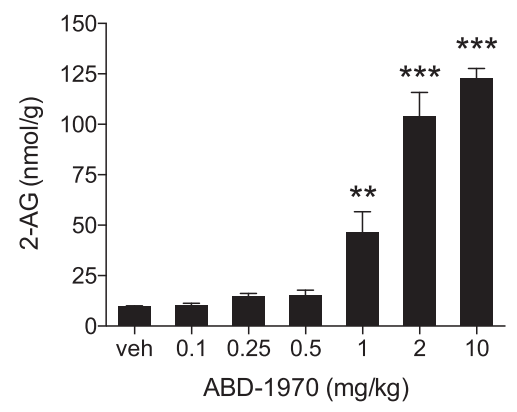

E

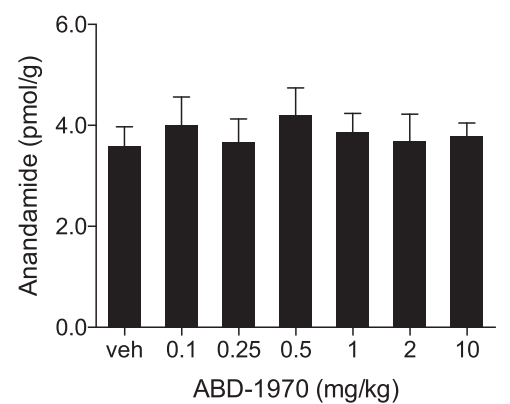

H

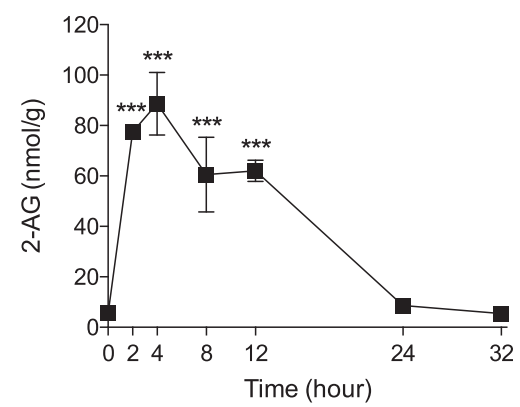

Fig. 4. ABD-1970 selectively inhibits MGLL in vivo and modulates brain levels of 2-AG, AA, and prostanoids. (A and B) ABD-1970 administration $(0.1-10 \mathrm{mg} / \mathrm{kg}$, p.o., 4 hours postdose, $0.5 \%$ methylcellulose vehicle, $n=5$ rats per group) resulted in selective inhibition of MGLL in the brain (A), with an $\mathrm{ED}_{50}$ value of $0.7 \mathrm{mg} / \mathrm{kg}(\mathrm{B})$. (C-E) Dose-dependent increases in brain 2-AG (C) and reductions in brain AA and the prostaglandins PGE $\mathrm{B}_{2}$ and PGD (D) $_{2}$ were observed without alterations in brain anandamide concentrations $(\mathrm{E})$. (F-H) Concentrations of ABD-1970 in the brain and blood (F), inhibition of brain MGLL activity (G), and increased 2-AG concentrations were observed after a single oral dose of $12 \mathrm{mg} / \mathrm{kg} \mathrm{ABD}-1970$ (p.o., $n=3$ rats per time point, 7:2:1 PEG400/ethanol/PBS vehicle) (H). Data represent the mean \pm S.E.M. $* P<0.05 ; * * P<0.01 ; * * * P<0.001$ vs. vehicle $(\mathrm{C}$ and $\mathrm{D})$ or at 0 hours $(\mathrm{G}$ and H). $\alpha / \beta$ hydrolase domain containing 6 FAAH, fatty acid amide hydrolase; MGLL, monoacylglycerol lipase PEG400, polyethylene glycol 400; PGD ${ }_{2}$, prostaglandin D2. 6k-PGF1a, 6-ketoprostaglandin F1a; $\mathrm{PGD}_{2}$, prostaglandin D2; $\mathrm{PGE}_{2}$, prostaglandin E2; PGF2a, prostaglandin F2a

inhibited MGLL in the brain, with an $\mathrm{ED}_{50}$ of $0.7 \mathrm{mg} / \mathrm{kg}$ at 4 hours postdose (Fig. 4, A and B). ABD-1970 administration was selective for MGLL among rat brain serine hydrolases with only one additional target identified (ABHD6), which was partially inhibited at the highest dose tested of $10 \mathrm{mg} / \mathrm{kg}$ (Fig. 4A). Significant increases in brain 2-AG concentrations were observed at a dose of $1 \mathrm{mg} / \mathrm{kg}$ ABD-1970, near the $\mathrm{ED}_{50}$, with $>100 \mathrm{nmol} / \mathrm{g} 2$-AG observed after single doses of 2 or $10 \mathrm{mg} / \mathrm{kg}$ (Fig. 4C). As has been previously reported in mice treated with MGLL inhibitors (Nomura et al., 2011), reductions in brain $\mathrm{AA}$ and the prostanoids prostaglandin $\mathrm{E} 2\left(\mathrm{PGE}_{2}\right) /$ prostaglandin D2 were observed after ABD-1970 administration (Fig. 4D). Rat brain concentrations of the other major endocannabinoid anandamide were not affected (Fig. 4E).

The time-course effects of ABD-1970 were determined in rats after a single oral dose of $12 \mathrm{mg} / \mathrm{kg}$. Exposure to
ABD-1970 in the brain and blood (Fig. 4F) was associated with MGLL inhibition in the brain (Fig. 4G) and increases in brain 2-AG concentrations (Fig. 4H) for up to 12 hours postdose. Partial recovery of MGLL activity and normalization of brain 2-AG concentrations were observed by 24 hours postdose.

Similar dose- and time-dependent inhibition of MGLL and accumulation of 2-AG were observed after single oral doses of ABD-1970 in mice (Supplemental Fig. 3).

\section{Efficacy of ABD-1970 in the Rat Formalin Model of Acute Pain Correlates with PK and CNS Biomarkers and Potentiates Analgesic Standards of Care}

MGLL inhibitors produce antihyperalgesic and antiallodynic effects in multiple rodent models of pain (Grabner et al., 2017). To understand the relationship between brain and blood ABD-1970 concentrations, brain MGLL target 
A

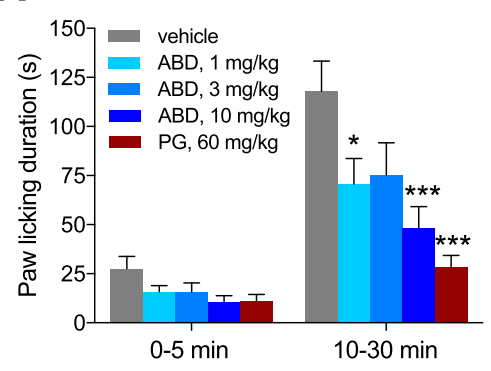

C

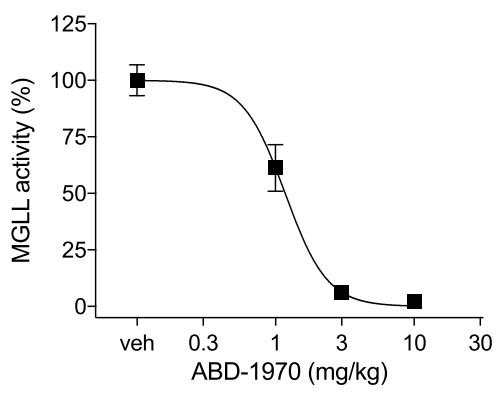

E

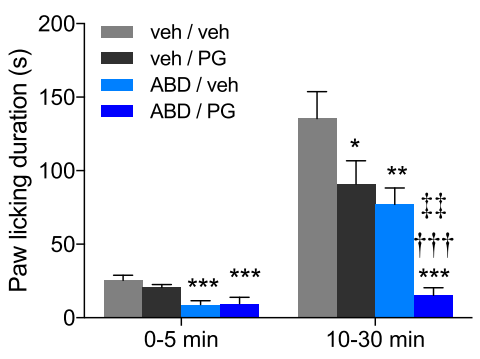

B

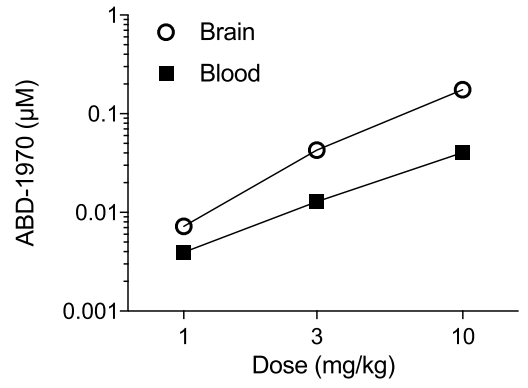

D

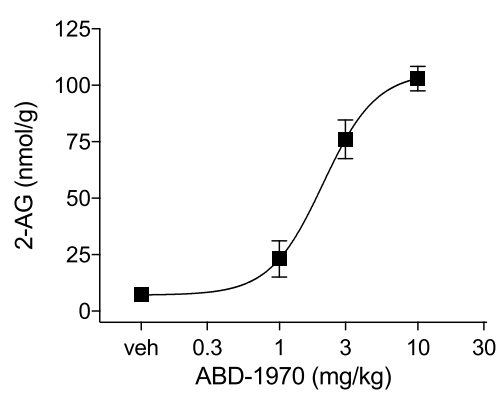

F

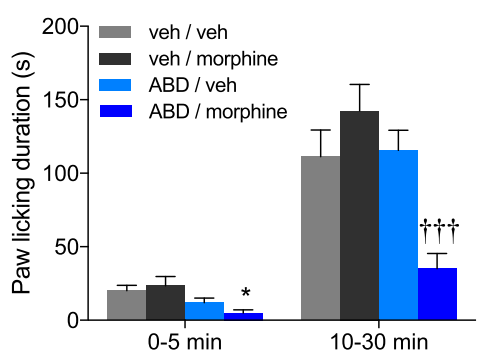

Fig. 5. Efficacy of ABD-1970 in the rat formalin model correlates with PK and CNS biomarkers and potentiates analgesic standards of care. (A) Single doses of ABD-1970 (1-10 mg/kg, p.o.) or the positive control pregabalin $(60 \mathrm{mg} /$ $\mathrm{kg}$, p.o.) administered 4 hours prior to formalin $(2.5 \%, 50 \mu \mathrm{l}$, intraplantar injection) reduced pain behavior measured as total paw licking duration $\left(n=10\right.$ rats per group; ${ }^{*} P<0.05$; $* * * P<0.001$ vs. vehicle). (B-D) Blood and brain concentrations of ABD-1970 (B) measured at the end of the formalin test (approximately 4.75 hours postdose, $n=3-10$ rats per group) were inversely associated with brain MGLL activity (C) and positively associated with increased 2-AG concentrations (D). (E) The combination of ABD-1970 (2 mg/ $\mathrm{kg}$, p.o., 4 hours pretest) with pregabalin $(10 \mathrm{mg} / \mathrm{kg}$, p.o., 4 hours pretest) resulted in enhanced antinociceptive activity compared with either agent individually $(n=8-10$ rats per group; $* P<0.05 ; * * P<0.01 ; * * * P<0.001$ vs. vehicle; $P<0.01$ vs. ABD-1970; ${ }^{\dagger} P<0.001$ vs. pregabalin). (F) The combination of ABD-1970 $(1 \mathrm{mg} / \mathrm{kg}$, p.o., 4 hours pretest) with morphine $(2.49 \mathrm{mg} / \mathrm{kg}$, s.c., 0.5 hours pretest) also resulted in enhanced antinociceptive activity compared with either agent individually $(n=9$ to 10 rats per group; $* P<0.05$ vs. vehicle; ${ }^{\dagger \dagger} P<0.0001$ vs. morphine). Data represent the mean \pm S.E.M. ABD, ABD1970; PG, pregabalin; veh, vehicle. engagement, brain 2-AG concentrations, and antinociceptive efficacy, a $\mathrm{PK} / \mathrm{PD}$ relationship was described in the rat formalin model (Tjølsen et al., 1992). Formalin administration to the rat hind paw results in spontaneous pain behaviors, including paw licking, which was quantified using automated behavior tracking cages. Single oral doses of ABD-1970 produced robust antinociceptive activity in formalin-challenged rats (Fig. 5A), with an $\mathrm{ED}_{50}$ of $1 \mathrm{mg} / \mathrm{kg}$ calculated for latephase (10-30 minutes postformalin) paw licking duration. The reductions in late-phase pain behavior at the $\mathrm{ED}_{50}$ were associated with ABD-1970 !concentrations of $4 \mathrm{nM}$ in the blood and $7 \mathrm{nM}$ in the brain (Fig. 5B), 40\% inhibition of MGLL in the brain (Fig. 5C), and 3-fold increases in brain 2-AG concentrations (Fig. 5D).

MGLL inhibitors have been shown to enhance the antinociceptive effects of analgesic drugs in preclinical models of pain (Crowe et al., 2015, 2017; Wilkerson et al., 2016). We evaluated the effects of ABD-1970 in combination with the $\alpha-2$, $\delta$-1 calcium channel blocker pregabalin or the $\mu$ opioid receptor agonist morphine in the rat formalin model. Coadministration of ABD-1970 and pregabalin significantly reduced paw licking pain behavior in formalin-treated rats to a greater extent than either agent individually (Fig. 5E). Treatment of rats with ABD-1970 and morphine similarly reduced formalin responses, whereas administration of either compound alone lacked efficacy in this experiment (Fig. 5F). These results demonstrate that MGLL inhibition potentiates the antinociceptive effects of mechanistically distinct and clinically effective analgesics.

\section{ABD-1970 Demonstrates Broad-Spectrum Antinociceptive and Antipruritic Effects without Cannabimimetic Effects on Locomotor Activity}

ABD-1970 was tested in additional preclinical models of pain of distinct pathologic origins and in a model of pruritus. In the inflammatory pain model caused by plantar injection of CFA, ABD-1970 administration produced dose-dependent antiallodynic effects for up to 6 hours postdose, with a calculated $\mathrm{ED}_{50}$ value of $2 \mathrm{mg} / \mathrm{kg}$ at 4 hours postdose (Fig. 6A). A single dose of $20 \mathrm{mg} / \mathrm{kg}$ ABD-1970 reduced mechanical allodynia in rats caused by plantar incision to a similar extent as a nonsedative dose of the opioid buprenorphine (Fig. 6B). In the CCI model of neuropathic pain, a single dose of $30 \mathrm{mg} / \mathrm{kg}$ ABD-1970 or pregabalin significantly reversed allodynic responses compared with vehicle-treated rats (Fig. 6C). In a rat model of pruritus induced by intradermal injection of serotonin, ABD-1970 demonstrated significant antipruritic effects at doses as low at $1 \mathrm{mg} / \mathrm{kg}$ that were similar to that of the opioid antagonist naltrexone (Fig. 6D).

The antinociceptive and antipruritic effects observed after ABD-1970 treatment occurred without overt behavioral 


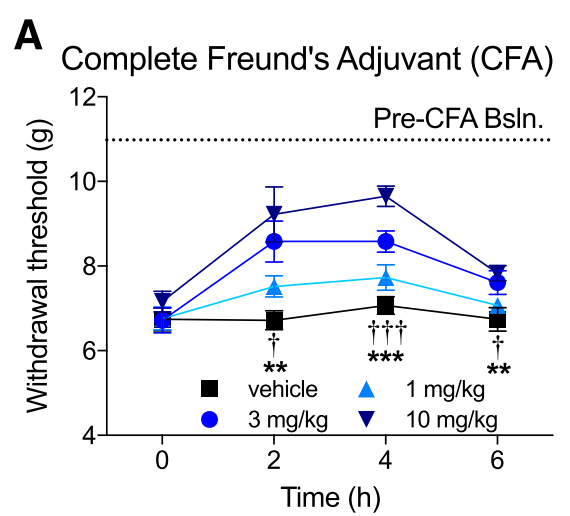

C

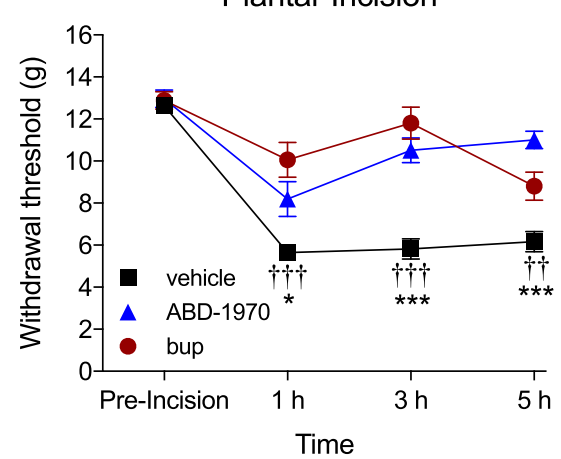

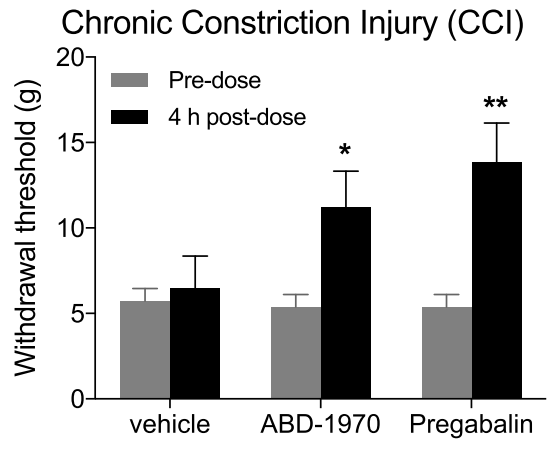

D

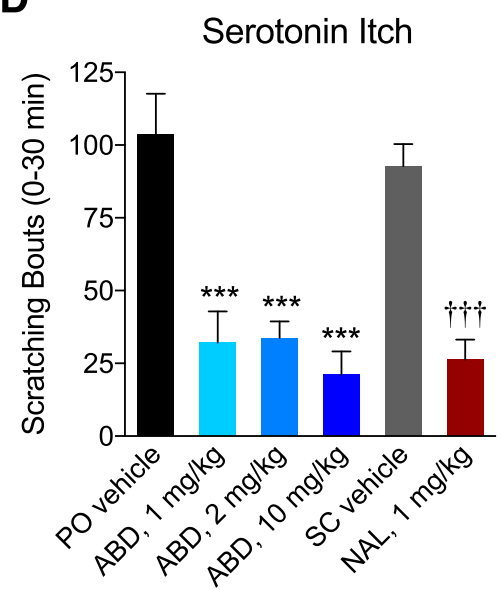

E

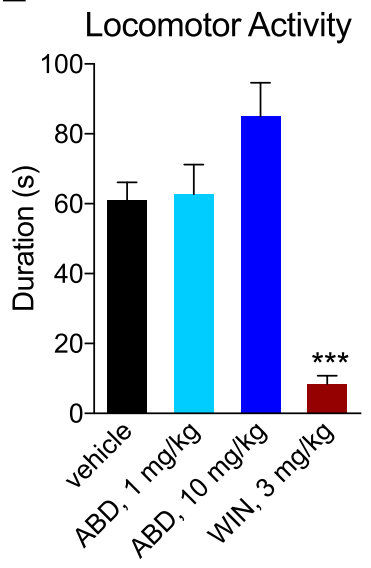

F

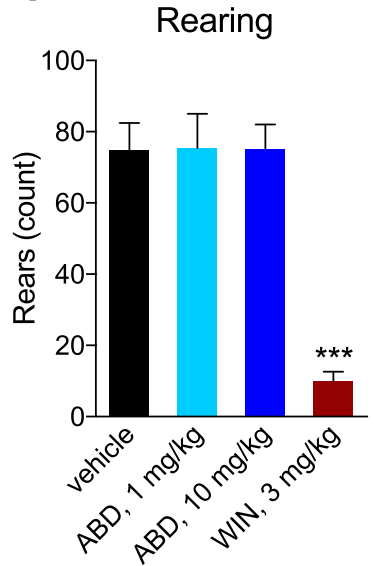

G

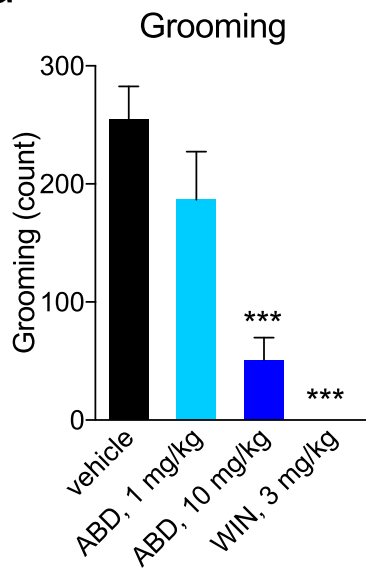

Fig. 6. ABD-1970 demonstrates broad-spectrum antinociceptive and antipruritic effects without cannabimimetic effects on locomotor activity. (A-C) Antinociceptive effects of ABD-1970 (p.o., 0.5\% methylcellulose vehicle) was demonstrated in the following models: (A) CFA model of inflammatory pain $\left(n=8\right.$ rats per group; $* * P<0.01$ vs. $10 \mathrm{mg} / \mathrm{kg}$ ABD- $1970 ; * * * P<0.001$ vs. $10 \mathrm{mg} / \mathrm{kg}$ ABD- $1970 ;{ }^{\dagger} P<0.05 \mathrm{vs} .3 \mathrm{mg} / \mathrm{kg} \mathrm{ABD}-1970 ;{ }^{\dagger \dagger \dagger} P<0.001 \mathrm{vs} .3 \mathrm{mg} / \mathrm{kg}$ ABD-1970), (B) the plantar incision model of acute pain $\left(n=8\right.$ rats per group; $* P<0.05$ vs. ABD-1970; $* * * P<0.001$ vs. ABD-1970; ${ }^{\dagger \dagger} P<0.01$ vs. buprenorphine; ${ }^{\dagger \dagger} P<0.001 \mathrm{vs}$. buprenorphine), and (C) the CCI model of neuropathic pain ( $n=8$ to 9 rats per group; $* P<0.05 ; * * P<0.01$ vs. predose). (D) Antipruritic effects ABD-1970 (p.o., 0.5\% methylcellulose, 4 hours pretest) and naltrexone (s.c., $0.9 \%$ saline vehicle, 0.25 hours pretest) in rats after intradermal serotonin administration $\left(n=8\right.$ rats per group; *** $P<0.001$ vs. p.o. vehicle; ${ }^{\dagger \dagger} P<0.001$ vs. s.c. vehicle). (E-G) Behavioral effects of ABD1970 (p.o., 4 hours pretest) or the direct CB1/2 agonist WIN 55,212-2 (s.c., $1 \%$ Tween 80/99\% saline vehicle, 0.25 hours pretest) on total locomotor duration $(\mathrm{E})$, rearing frequency $(\mathrm{F})$, or grooming $(\mathrm{G})\left(n=7\right.$ to 8 rats per group; ${ }^{* * *} P<0.001$ vs. vehicle). Data represent the mean $\pm \mathrm{S} . \mathrm{E} . \mathrm{M}$. ABD, ABD1970; Bup, buprenorphine; NAL, naltrexone; WIN, WIN 55,212-2.

effects. Single doses of 1 and $10 \mathrm{mg} / \mathrm{kg}$ ABD-1970 did not reduce locomotor duration in a novel environment (Fig. 6E) or rearing (Fig. 6F), but did produce dose-dependent reductions in grooming (Fig. 6G). In stark contrast, a single 3-mg/kg dose of the exogenous cannabinoid WIN 55,212-2 dramatically reduced locomotor activity, rearing, and grooming (Fig. 6, E-G).

\section{MGLL and COX Inhibition Differentially Affect Prostanoid Production in Human Cells}

The anatomically contextualized contribution of MGLL to prostanoid metabolism in mice (Nomura et al., 2011) motivated us to evaluate the effects of ABD-1970 in human cellular systems alongside the nonselective COX inhibitor, indomethacin, and selective COX2 inhibitor, rofecoxib. Human whole blood can be stimulated with LPS endotoxin or calcium ionophores to produce inflammatory prostanoids, which are strongly suppressed by COX inhibition (Brideau et al., 1996; Warner et al., 1999). ABD-1970 treatment of whole blood effectively inhibits MGLL activity in PBMCs $\left(\mathrm{IC}_{50}=62 \mathrm{nM}\right)$.
Therefore, human blood represents an appropriate system to evaluate the effects of MGLL inhibition on stimulated prostanoid production and compare them with the effects of COX inhibition.

Human whole blood from two donors was treated with LPS (30 ng/ml, $37^{\circ} \mathrm{C}$, approximately 24 hours) to stimulate prostanoid production. Pretreatment of LPS-stimulated whole blood with ABD-1970 (0.03-30 $\mu \mathrm{M}, 30$ minutes, $37^{\circ} \mathrm{C}$ ) increased plasma 2-AG by approximately 7 -fold (Fig. 7A) and decreased plasma AA by approximately 2 -fold (Fig. $7 \mathrm{~B})$, but did not affect $\mathrm{PGE}_{2}$ or thromboxane $\mathrm{B} 2\left(\mathrm{TXB}_{2}\right)$ concentrations (Fig. 7C). In contrast, pretreatment of whole blood with the NSAID indomethacin $(30 \mu \mathrm{M}, 30$ minutes, $37^{\circ} \mathrm{C}$ ) suppressed production of $\mathrm{PGE}_{2}$ and $\mathrm{TXB}_{2}$ by more than 10-fold. The selective COX2 inhibitor rofecoxib $(10 \mu \mathrm{M}$, 30 minutes, $37^{\circ} \mathrm{C}$ ) also suppressed $\mathrm{PGE}_{2}$ and, to a lesser degree, $\mathrm{TXB}_{2}$, consistent with the dominant role of COX1 in thromboxane production in blood (Brideau et al., 1996; Warner et al., 1999).

Stimulation of human whole blood with the calcium ionophore ionomycin $\left(30 \mu \mathrm{M}, 30\right.$ minutes, $\left.37^{\circ} \mathrm{C}\right)$ increased production of 
A

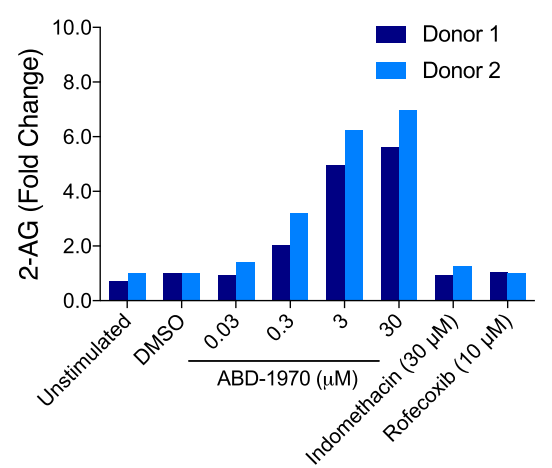

B Whole Blood (LPS-stimulated)

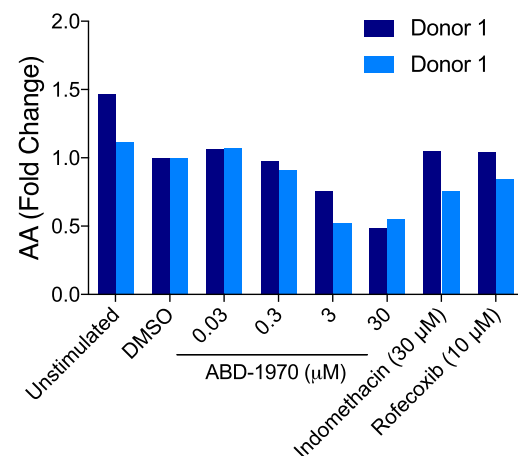

Whole Blood (lonomycin-stimulated)

D

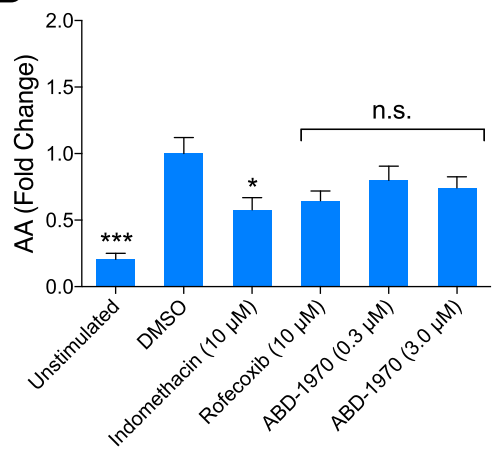

E

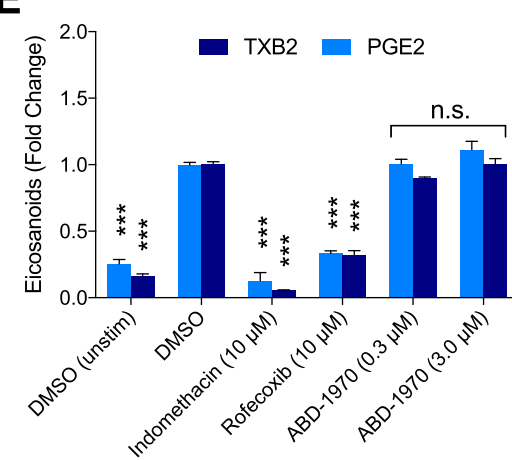

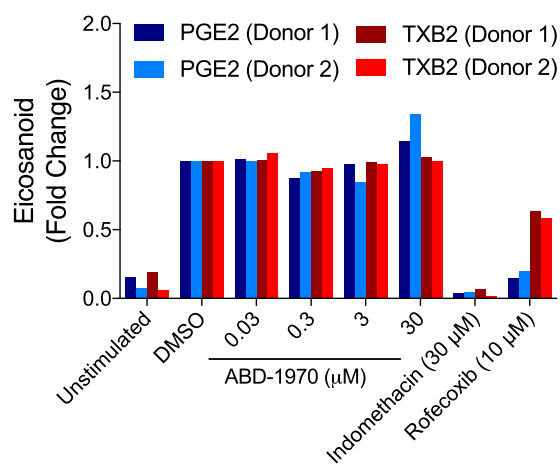

IL1 $\beta$-Stimulated HUVECs

$\mathbf{F}$

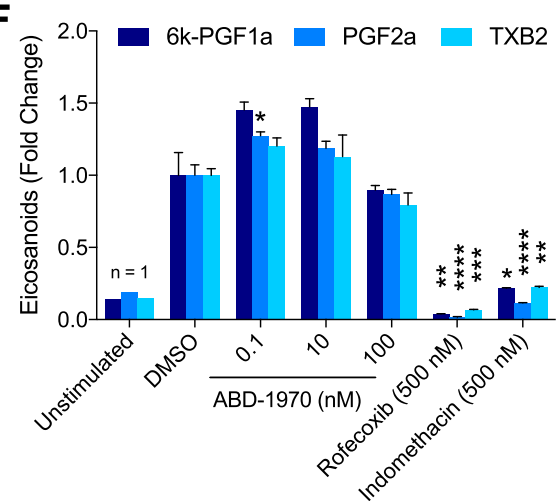

Fig. 7. MGLL and COX inhibition differentially affect prostanoid production in human cells. (A-F) The effects of ABD-1970, indomethacin, and rofecoxib on concentrations of 2-AG, AA, and prostanoid lipids were evaluated in human whole blood treated with LPS (A-C) or ionomycin (D and E) or primary HUVECs stimulated with IL-1 $\beta(\mathrm{F})$. In (A) through (C), data are from whole blood from two human donors. In (D) through (F), data are presented as the mean \pm S.E.M. $\left(n=3\right.$ per group; ${ }^{*} P<0.05 ; * * P<0.01 ; * * * P<0.001 ; * * * * P<0.0001$ vs. stimulated DMSO control). PGE 2 , prostaglandin $\mathrm{E} 2$; n.s., not significant; $\mathrm{TXB}_{2}$, thromboxane $\mathrm{B} 2$.

AA (Fig. 7D), and prostanoids ( $\mathrm{PGE}_{2}$ and $\mathrm{TXB}_{2}$ ) (Fig. 6E), as expected. Pretreatment of ionomycin-stimulated whole blood with ABD-1970 (0.3 and $3 \mu \mathrm{M})$ did not significantly alter plasma levels of AA (Fig. 7D) or of downstream prostanoid lipids (Fig. 7E). In contrast, indomethacin ( $10 \mu \mathrm{M}, 30$ minutes, $37^{\circ} \mathrm{C}$ ) suppressed production of $\mathrm{PGE}_{2}$ and $\mathrm{TXB}_{2}$ by 10 -fold or greater (Fig. $7 \mathrm{E})$, whereas rofecoxib $\left(10 \mu \mathrm{M}, 30\right.$ minutes, $\left.37^{\circ} \mathrm{C}\right)$ suppressed both $\mathrm{PGE}_{2}$ and $\mathrm{TXB}_{2}$ by approximately 3 -fold (Fig. 7E).

The impact of MGLL inhibition on prostanoid production in human endothelial cells was investigated in primary HUVECs stimulated with the inflammatory cytokine IL- $1 \beta$. HUVECs have previously been used to study the effect of COX inhibition on prostanoid production, as they generate important prostanoid lipids when stimulated (Niederberger et al., 2004; Salvado et al., 2009). These lipids include thromboxane $\mathrm{A}_{2}$ (measured as its stable breakdown product $\mathrm{TXB}_{2}$ ), which stimulates platelet aggregation and vasoconstriction, and prostacyclin (measured as its stable breakdown product 6-ketoprostaglandin $\mathrm{F} 1 \alpha$ ), which inhibits platelet aggregation and causes vasodilation (Salvado et al., 2009).

Stimulation of HUVECs with IL- $1 \beta$ increased the production of several prostanoid lipids, including 6-ketoprostaglandin $\mathrm{F} 1 \alpha, \mathrm{TXB}_{2}$, and prostaglandin F2 $\alpha$ (Fig. $7 \mathrm{~F}$ ). All of these prostanoids were suppressed when prestimulated HUVECs were treated with indomethacin $(500 \mathrm{nM})$ or rofecoxib $(500 \mathrm{nM})$, but not ABD-1970 (0.1-100 nM), despite confirmation of complete MGLL inhibition at 10 and $100 \mathrm{nM}$ ABD-1970 (Supplemental Fig. 4).

To investigate the functional consequences of MGLL inhibition on prostanoid signaling, ABD-1970 was tested for its effect on collagen-induced platelet aggregation, an assay in which NSAIDs display robust antiaggregatory activity (Armstrong et al., 2008). In human platelet-rich plasma, ABD-1970 (0.3-30 $\mu \mathrm{M})$ did not promote platelet aggregation or suppress aggregation induced by collagen (0\% agonist or antagonist activity at $0.3,3$, and $30 \mu \mathrm{M}$ ABD-1970). In contrast, indomethacin $(3 \mu \mathrm{M})$ suppressed collagen-induced aggregation by $98 \%$.

\section{Discussion}

Preparations of Cannabis and cannabinoid receptor agonists produce therapeutic effects in humans, which are accompanied by adverse CNS effects that limit the clinical dose. An alternative strategy to harness the clinical utility of cannabinoid receptor activation is to augment endocannabinoid signaling by limiting enzymatic degradation. MGLL is the major 2-AG hydrolase in rodents, and MGLL inhibitors produce a myriad of beneficial effects in animal models of pain, anxiety, epilepsy, traumatic brain injury, neuroinflammation, and neurodegeneration (Nomura et al., 2011; Chen et al., 2012; Piro et al., 2012; Griebel et al., 2015; Katz et al., 2015; von Rüden et al., 2015; Zhang et al., 2015; Sugaya et al., 2016; 
Grabner et al., 2017; Patel et al., 2017). To prepare for an MGLL inhibitor clinical program, we developed the selective MGLL inhibitor tool compound ABD-1970 to evaluate the consequences of MGLL inhibition in human tissues and cells and in rodent models of pain and pruritus.

ABD-1970 is a potent inhibitor of MGLL across species that acts via a time-dependent and irreversible mechanism, which is consistent with a covalent interaction, as has been observed for other mechanism-based carbamate-containing MGLL inhibitors (Long et al., 2009a; Chang et al., 2012; Niphakis et al., 2012; Griebel et al., 2015; Butler et al., 2017). Covalent inhibition can be an attractive mechanism for enzyme inactivation because high biochemical efficiency and an extended duration of target engagement, compared with the PK profile, can often be achieved (Johnson et al., 2010; Singh et al., 2011). The time-dependent and irreversible nature of the ABD-1970-MGLL interaction likely contributes to the high potency and sustained activity that ABD-1970 displays in vivo. Once MGLL is inhibited, ABD-1970 does not need to compete with rising 2-AG concentrations, which ultimately reach $>100 \mathrm{nmol} / \mathrm{g}$ (approximately $100 \mu \mathrm{M}$ ) in the brain, and the maintenance of inhibition is not solely determined by sustained compound exposure but instead is largely dependent on protein biosynthesis.

The exceptional potency and selectivity of ABD-1970 was achieved through a medicinal chemistry campaign that leveraged the chemical proteomics platform ABPP (Niphakis and Cravatt, 2014) to simultaneously evaluate and optimize these parameters (Cisar et al., 2018). Extensive ABPP profiling in human tissues and cells revealed that ABD-1970 has at least 77-fold selectivity toward MGLL over other serine hydrolases and completely inhibits MGLL at concentrations that are devoid of any serine hydrolase off-target interactions. Of the $>70$ serine hydrolase enzymes screened, many are wholly uncharacterized with regard to their substrates and functions; as such, substrate-agnostic techniques like ABPP represent the only way to functionally evaluate these enzymes and confirm selectivity.

The favorable pharmacological profile demonstrated by ABD-1970 in vitro was maintained in vivo after oral administration to mice and rats, as ABD-1970 selectively and sustainably inhibited MGLL in the brain with concomitant increases in whole brain 2-AG concentrations. Single doses of ABD-1970 effectively inhibited brain MGLL activity in both species, with $\mathrm{ED}_{50}$ values of approximately $1 \mathrm{mg} / \mathrm{kg}$. The doseresponse relationship revealed marked sensitivity of brain 2-AG to MGLL activity because partial inhibition of MGLL in the brain was sufficient to elevate 2-AG levels, which ultimately increased by $>10$-fold once MGLL was completely inhibited. In contrast, in the rat, approximately 2 -fold reductions in levels of $\mathrm{AA}$ and $\mathrm{PGE}_{2}$ /prostaglandin $\mathrm{D}_{2}$ in the brain were associated with near-complete inhibition of MGLL.

To understand the relationship between ABD-1970 PK, MGLL target engagement, 2-AG, and antinociceptive efficacy, we used the rat formalin pain model. This model provided the opportunity to evaluate the effects of ABD-1970 administration on two distinct nociceptive modalities: 1) an early phase corresponding to direct activation of peripheral sensory nerves and 2) a late phase corresponding largely to central sensitization (Tjølsen et al., 1992). Single doses of ABD-1970 numerically reduced pain behavior in the early phase and significantly reduced pain behaviors in the late phase of the formalin response, with an $\mathrm{ED}_{50}$ of $1 \mathrm{mg} / \mathrm{kg}$. The antinociceptive effects observed at the $\mathrm{ED}_{50}$ were associated with $\mathrm{ABD}$ 1970 concentrations less than $10 \mathrm{nM}$ in the brain and blood, $40 \%$ inhibition of brain MGLL activity, and 3-fold elevations in brain 2-AG concentrations. Multiple previous studies have demonstrated sustained antinociceptive, antiallodynic, and antihyperalgesic effects after repeated administration of MGLL inhibitors at low doses that partially inhibit MGLL in the brain (Busquets-Garcia et al., 2011; Kinsey et al., 2013; Burston et al., 2016; Curry et al., 2018), whereas tolerance to these effects has been observed after prolonged, complete MGLL inhibition or genetic ablation (Chanda et al., 2010; Schlosburg et al., 2010; Ignatowska-Jankowska et al., 2014). The functional tolerance observed with complete MGLL inactivation and maximal elevation of brain 2-AG concentrations is associated with downregulation and/or desensitization of brain CB1 receptors (Chanda et al., 2010; Schlosburg et al., 2010; Navia-Paldanius et al., 2015). The ABD-1970 PK/PD relationship in the rat formalin model revealed that painmodifying activity was observed at low doses and exposures of ABD-1970 that only partially inhibited brain MGLL and caused submaximal elevations of $2-\mathrm{AG}$ in the brain. Therefore, low doses of ABD-1970 near the $\mathrm{ED}_{50}$ of $1 \mathrm{mg} / \mathrm{kg}$ would be predicted to maintain efficacy after repeated dosing.

In addition to demonstrating significant antinociceptive activity as a single agent in the rat formalin model, ABD1970 also potentiated the activity of two analgesic standards of care: pregabalin and morphine; this is in agreement with the synergistic activity that has been shown previously for MGLL inhibitors and similar agents in other pain models (Wilkerson et al., 2016; Crowe et al., 2017). That MGLL inhibition enhanced the efficacy of these clinically effective drugs suggests that in addition to providing pain relief as a single agent, an MGLL inhibitor may find clinical utility in enhancing the effectiveness of non-narcotic pain killers like pregabalin or limiting the dose needed of opioid drugs to provide adequate pain relief. The effects of ABD-1970 on pain were not limited to acute pain caused by formalin; antinociceptive or antiallodynic effects were observed in rat models of postoperative pain, neuropathic pain, and inflammatory pain. ABD-1970 also significantly reduced itch behavior in the rat serotonin model of pruritus, consistent with previously published reports with MGLL inhibitors in mice (Tosun et al., 2015; Yesilyurt et al., 2016). These effects occurred without obvious cannabimimetic effects or sedation, as ABD-1970 did not reproduce the behavioral effects of the direct cannabinoid receptor agonist WIN 55,212-2 in a novel open field. ABD-1970 did dose-dependently reduce grooming behavior in the novel environment, which may be related to the antipruritic and anxiolytic effects of this mechanism.

Selective MGLL inhibition by ABD-1970 in the rodent brain effectively increased total brain 2-AG concentrations, as has been previously demonstrated after pharmacological or genetic inactivation of MGLL (Long et al., 2009a; Schlosburg et al., 2010). Experiments with ABD-1970 in human tissues and cells suggest that MGLL is likely to similarly regulate 2-AG in humans. ABD-1970 treatment of human brain tissue reduced $2-A G$ hydrolysis by $>95 \%$, which is comparable to the approximately $85 \%$ of the total 2-AG hydrolase activity mediated by MGLL in mouse brain preparations (Blankman et al., 2007), which therefore increases confidence that MGLL inhibition in the human brain will amplify 2 -AG signaling and 
produce the therapeutically beneficial effects demonstrated in preclinical studies. Likewise, treatment of human whole blood with ABD-1970 inhibited MGLL in blood cells and elevated plasma 2-AG concentrations. The concentration-dependent inhibition of MGLL observed in PBMCs after treatment of whole blood provides a viable clinical biomarker strategy to measure peripheral MGLL target engagement in human subjects or patients administered an MGLL inhibitor.

In rodents, MGLL serves as a source of AA for COXmediated prostanoid generation in a subset of tissues (Nomura et al., 2011). Here, we used ABD-1970 to evaluate the contribution of MGLL to the generation of AA-derived prostanoids in human whole blood and endothelial cells. The nonselective COX inhibitor indomethacin and the selective COX2 inhibitor rofecoxib were evaluated in parallel to compare the effects of MGLL and COX inhibition in assays known to be sensitive to COX inhibitors. MGLL inhibition did not suppress stimulated prostanoid production or platelet aggregation and was therefore differentiated from COX inhibition. Our results show that despite their involvement in sequential steps of the AA metabolic pathway, the effects of inhibiting MGLL or COX enzymes in human cells are distinct, suggesting that MGLL inhibitors are unlikely to replicate the toxicity profiles displayed by COX inhibitors.

In conclusion, ABD-1970 is a highly potent, selective, and orally bioavailable MGLL inhibitor, which we have used to evaluate the effects of MGLL inhibition in human tissues and cells and in rodent models of pain and itch. These results support use of ABD-1970 as a tool MGLL inhibitor in preclinical models and further clinical exploration of the mechanism. Toward this end, we have developed a mechanistically similar MGLL inhibitor that is currently in clinical development for neurologic indications, including Tourette syndrome, spasticity in multiple sclerosis, and pain (Cisar et al., 2018).

\section{Acknowledgments}

We thank Dr. Guy Kennett (Saretius Ltd.) for helpful discussions and insight into the formalin pain model, as well as Dr. Clare Doris and Dr. Hayley Gooding (Aquila BioMedical) and Dr. Jenny Wilkerson and Aron Lichtman (Virginia Commonwealth University) for assistance with the CCI model. We also thank Dr. Nathalie C. Franc (Franc Consulting, San Diego, CA) for providing medical writing support (editing and formatting). Finally, we thank Ben Cravatt (The Scripps Research Institute) for critical reading of the manuscript.

\section{Authorship Contributions}

Participated in research design: Clapper, Fraser, Ezekowitz, O’Neill, Blankman.

Conducted experiments: Clapper, Henry, Niphakis, Knize, Coppola, Simon, Ngo, R. A. Herbst, D. M. Herbst, Reed, Viader, Alexander, Cunningham, Blankman.

Contributed new reagents or analytic tools: Cisar, Weber, Jones, Grice.

Performed data analysis: Clapper, Niphakis, Simon, Viader, Alexander, Cunningham, Blankman.

Wrote or contributed to the writing of the manuscript: Clapper, Henry, Niphakis, Knize, Cisar, O’Neill, Blankman.

\section{References}

Abbott FV, Franklin KB, and Westbrook RF (1995) The formalin test: scoring properties of the first and second phases of the pain response in rats. Pain 60:91-102. Armstrong PC, Truss NJ, Ali FY, Dhanji AA, Vojnovic I, Zain ZN, Bishop-Bailey D, Paul-Clark MJ, Tucker AT, Mitchell JA, et al. (2008) Aspirin and the in vitro linear relationship between thromboxane A2-mediated platelet aggregation and platelet production of thromboxane A2. J Thromb Haemost 6:1933-1943.
Bar-On P, Millard CB, Harel M, Dvir H, Enz A, Sussman JL, and Silman I (2002) Kinetic and structural studies on the interaction of cholinesterases with the antiAlzheimer drug rivastigmine. Biochemistry 41:3555-3564.

Bedse G, Bluett RJ, Patrick TA, Romness NK, Gaulden AD, Kingsley PJ, Plath N, Marnett LJ, and Patel S (2018) Therapeutic endocannabinoid augmentation for mood and anxiety disorders: comparative profiling of FAAH, MAGL and dual inhibitors. Transl Psychiatry 8:92.

Bennett GJ and Xie YK (1988) A peripheral mononeuropathy in rat that produces disorders of pain sensation like those seen in man. Pain 33:87-107.

Berry LM, Wollenberg L, and Zhao Z (2009) Esterase activities in the blood, liver and intestine of several preclinical species and humans. Drug Metab Lett 3:70-77.

Blankman JL, Simon GM, and Cravatt BF (2007) A comprehensive profile of brain enzymes that hydrolyze the endocannabinoid 2-arachidonoylglycerol. Chem Biol 14:1347-1356.

Boersema PJ, Raijmakers R, Lemeer S, Mohammed S, and Heck AJ (2009) Multiplex peptide stable isotope dimethyl labeling for quantitative proteomics. Nat Protoc 4 484-494.

Born GV (1962) Aggregation of blood platelets by adenosine diphosphate and its reversal. Nature 194:927-929.

Brennan TJ, Vandermeulen EP, and Gebhart GF (1996) Characterization of a rat model of incisional pain. Pain 64:493-501.

Brideau C, Kargman S, Liu S, Dallob AL, Ehrich EW, Rodger IW, and Chan CC (1996) A human whole blood assay for clinical evaluation of biochemical efficacy of cyclooxygenase inhibitors. Inflamm Res 45:68-74.

Burston JJ, Mapp PI, Sarmad S, Barrett DA, Niphakis MJ, Cravatt BF, Walsh DA, and Chapman V (2016) Robust anti-nociceptive effects of monoacylglycerol lipase inhibition in a model of osteoarthritis pain. Br J Pharmacol 173:3134-3144.

Busquets-Garcia A, Puighermanal E, Pastor A, de la Torre R, Maldonado R, and Ozaita A (2011) Differential role of anandamide and 2-arachidonoylglycerol in memory and anxiety-like responses. Biol Psychiatry 70:479-486.

Butler CR, Beck EM, Harris A, Huang Z, McAllister LA, Am Ende CW, Fennell K, Foley TL, Fonseca K, Hawrylik SJ, et al. (2017) Azetidine and piperidine carbamates as efficient, covalent inhibitors of monoacylglycerol lipase. J Med Chem 60: 9860-9873.

Chanda PK, Gao Y, Mark L, Btesh J, Strassle BW, Lu P, Piesla MJ, Zhang MY, Bingham B, Uveges A, et al. (2010) Monoacylglycerol lipase activity is a critical modulator of the tone and integrity of the endocannabinoid system. Mol Pharmacol 78:996-1003.

Chang JW, Cognetta AB, 3rd, Niphakis MJ, and Cravatt BF (2013) Proteome-wide reactivity profiling identifies diverse carbamate chemotypes tuned for serine hydrolase inhibition. ACS Chem Biol 8:1590-1599.

Chang JW, Niphakis MJ, Lum KM, Cognetta AB, 3rd, Wang C, Matthews ML, Niessen S, Buczynski MW, Parsons LH, and Cravatt BF (2012) Highly selective inhibitors of monoacylglycerol lipase bearing a reactive group that is bioisosteric with endocannabinoid substrates. Chem Biol 19:579-588.

Chen R, Zhang J, Wu Y, Wang D, Feng G, Tang Y-P, Teng Z, and Chen C (2012) Monoacylglycerol lipase is a therapeutic target for Alzheimer's disease. Cell Rep 2: 1329-1339.

Cisar JS, Weber OD, Clapper JR, Blankman JL, Henry CL, Simon GM, Alexander JP, Jones TK, Ezekowitz RAB, O'Neill GP, et al. (2018). Identification of ABX-1431, a selective inhibitor of monoacylglycerol lipase and clinical candidate for treatment of neurological disorders. J Med Chem DOI: 10.1021/acs.jmedchem.8b00951 [published ahead of print].

Cociorva D, Tabb DL, and Yates JR (2007) Validation of tandem mass spectrometry database search results using DTASelect. Curr Protoc Bioinformatics 16: 13.4.1-13.4.14. doi:10.1002/0471250953.bi1304s16.

Copeland RA (2000). Enzymes: A Practical Introduction to Structure, Mechanism, and Data Analysis, 2nd ed, Wiley-VCH, New York.

Crowe MS and Kinsey SG (2017) MAGL inhibition modulates gastric secretion and motility following NSAID exposure in mice. Eur J Pharmacol 807:198-204.

Crowe MS, Leishman E, Banks ML, Gujjar R, Mahadevan A, Bradshaw HB, and Kinsey SG (2015) Combined inhibition of monoacylglycerol lipase and cyclooxygenases synergistically reduces neuropathic pain in mice. Br J Pharmacol 172:1700-1712.

Crowe MS, Wilson CD, Leishman E, Prather PL, Bradshaw HB, Banks ML, and Kinsey SG (2017) The monoacylglycerol lipase inhibitor KML29 with gabapentin synergistically produces analgesia in mice. $\mathrm{Br} J$ Pharmacol 174:4523-4539.

Curry ZA, Wilkerson JL, Bagdas D, Kyte SL, Patel N, Donvito G, Mustafa MA, Poklis JL, Niphakis MJ, Hsu KL, et al. (2018) Monoacylglycerol lipase inhibitors reverse paclitaxel-induced nociceptive behavior and proinflammatory markers in a mouse model of chemotherapy-induced neuropathy. J Pharmacol Exp Ther 366:169-183.

Funk CD (2001) Prostaglandins and leukotrienes: advances in eicosanoid biology. Science 294:1871-1875.

Grabner GF, Zimmermann R, Schicho R, and Taschler U (2017) Monoglyceride lipase as a drug target: at the crossroads of arachidonic acid metabolism and endocannabinoid signaling. Pharmacol Ther 175:35-46.

Griebel G, Pichat P, Beeské S, Leroy T, Redon N, Jacquet A, Françon D, Bert L, Even L, Lopez-Grancha M, et al. (2015) Selective blockade of the hydrolysis of the endocannabinoid 2-arachidonoylglycerol impairs learning and memory performance while producing antinociceptive activity in rodents. Sci Rep 5:7642.

Gulyas AI, Cravatt BF, Bracey MH, Dinh TP, Piomelli D, Boscia F, and Freund TF (2004) Segregation of two endocannabinoid-hydrolyzing enzymes into pre- and postsynaptic compartments in the rat hippocampus, cerebellum and amygdala. Eur J Neurosci 20:441-458.

Hsu K-L, Tsuboi K, Adibekian A, Pugh H, Masuda K, and Cravatt BF (2012) DAGL $\beta$ inhibition perturbs a lipid network involved in macrophage inflammatory responses. Nat Chem Biol 8:999-1007.

Ignatowska-Jankowska B, Wilkerson JL, Mustafa M, Abdullah R, Niphakis M, Wiley JL, Cravatt BF, and Lichtman AH (2015) Selective monoacylglycerol lipase inhibitors: antinociceptive versus cannabimimetic effects in mice. $J$ Pharmacol Exp Ther 353:424-432. 
Ignatowska-Jankowska BM, Ghosh S, Crowe MS, Kinsey SG, Niphakis MJ, Abdullah RA, Tao Q, O' Neal ST, Walentiny DM, Wiley JL, et al. (2014) In vivo characterization of the highly selective monoacylglycerol lipase inhibitor KML29: antinociceptive activity without cannabimimetic side effects. $\mathrm{Br} J$ Pharmacol 171: 1392-1407

Jessani N, Niessen S, Wei BQ, Nicolau M, Humphrey M, Ji Y, Han W, Noh DY, Yates JR, 3rd, Jeffrey SS, et al. (2005) A streamlined platform for high-content functional proteomics of primary human specimens. Nat Methods 2:691-697.

Johnson DS, Weerapana E, and Cravatt BF (2010) Strategies for discovering and derisking covalent, irreversible enzyme inhibitors. Future Med Chem 2:949-964.

Kano M, Ohno-Shosaku T, Hashimotodani Y, Uchigashima M, and Watanabe M (2009) Endocannabinoid-mediated control of synaptic transmission. Physiol Rev 89:309-380.

Katona I and Freund TF (2008) Endocannabinoid signaling as a synaptic circuit breaker in neurological disease. Nat Med 14:923-930.

Katona I, Urbán GM, Wallace M, Ledent C, Jung KM, Piomelli D, Mackie K, and Freund TF (2006) Molecular composition of the endocannabinoid system at glutamatergic synapses. J Neurosci 26:5628-5637.

Katz PS, Sulzer JK, Impastato RA, Teng SX, Rogers EK, and Molina PE (2015) Endocannabinoid degradation inhibition improves neurobehavioral function, blood-brain barrier integrity, and neuroinflammation following mild traumatic brain injury. J Neurotrauma 32:297-306.

Keith JM, Jones WM, Tichenor M, Liu J, Seierstad M, Palmer JA, Webb M, Karbarz M, Scott BP, Wilson SJ, et al. (2015) Preclinical characterization of the FAAH inhibitor JNJ-42165279. ACS Med Chem Lett 6:1204-1208.

Kinsey SG, Nomura DK, O’Neal ST, Long JZ, Mahadevan A, Cravatt BF, Grider JR, and Lichtman AH (2011) Inhibition of monoacylglycerol lipase attenuates nonsteroidal anti-inflammatory drug-induced gastric hemorrhages in mice. J Pharmacol Exp Ther 338:795-802.

Kinsey SG, Wise LE, Ramesh D, Abdullah R, Selley DE, Cravatt BF, and Lichtman $\mathrm{AH}$ (2013) Repeated low-dose administration of the monoacylglycerol lipase inhibitor JZL184 retains cannabinoid receptor type 1-mediated antinociceptive and gastroprotective effects. J Pharmacol Exp Ther 345:492-501.

Leung D, Hardouin C, Boger DL, and Cravatt BF (2003) Discovering potent and selective reversible inhibitors of enzymes in complex proteomes. Nat Biotechnol 21 687-691.

Long JZ, Li W, Booker L, Burston JJ, Kinsey SG, Schlosburg JE, Pavón FJ, Serrano AM, Selley DE, Parsons LH, et al. (2009a) Selective blockade of 2-arachidonoylglycerol hydrolysis produces cannabinoid behavioral effects. Nat Chem Biol 5:37-44.

Long JZ, Nomura DK, and Cravatt BF (2009b) Characterization of monoacylglycerol lipase inhibition reveals differences in central and peripheral endocannabinoid metabolism. Chem Biol 16:744-753.

Mechoulam R, Ben-Shabat S, Hanus L, Ligumsky M, Kaminski NE, Schatz AR, Gopher A, Almog S, Martin BR, Compton DR, et al. (1995) Identification of an endogenous 2-monoglyceride, present in canine gut, that binds to cannabinoid receptors. Biochem Pharmacol 50:83-90.

Navia-Paldanius D, Aaltonen N, Lehtonen M, Savinainen JR, Taschler U, Radner FP, Zimmermann R, and Laitinen JT (2015) Increased tonic cannabinoid CB1R activity and brain region-specific desensitization of $\mathrm{CB} 1 \mathrm{R}$ Gi/o signaling axis in mice with global genetic knockout of monoacylglycerol lipase. Eur J Pharm Sci $\mathbf{7 7}$ 180-188.

Niederberger E, Manderscheid C, Grösch S, Schmidt H, Ehnert C, and Geisslinger G (2004) Effects of the selective COX-2 inhibitors celecoxib and rofecoxib on human vascular cells. Biochem Pharmacol 68:341-350

Niphakis MJ and Cravatt BF (2014) Enzyme inhibitor discovery by activity-based protein profiling. Annu Rev Biochem 83:341-377.

Niphakis MJ, Johnson DS, Ballard TE, Stiff C, and Cravatt BF (2012) Ohydroxyacetamide carbamates as a highly potent and selective class of endocannabinoid hydrolase inhibitors. ACS Chem Neurosci 3:418-426.

Nomura DK, Morrison BE, Blankman JL, Long JZ, Kinsey SG, Marcondes MCG, Ward AM, Hahn YK, Lichtman AH, Conti B, et al. (2011) Endocannabinoid hydrolysis generates brain prostaglandins that promote neuroinflammation. Science 334:809-813.

Patel S, Hill MN, Cheer JF, Wotjak CT, and Holmes A (2017) The endocannabinoid system as a target for novel anxiolytic drugs. Neurosci Biobehav Rev 76 (Pt A): $56-66$.

Patricelli MP, Giang DK, Stamp LM, and Burbaum JJ (2001) Direct visualization of serine hydrolase activities in complex proteomes using fluorescent active sitedirected probes. Proteomics 1:1067-1071.
Pertwee RG (2012) Targeting the endocannabinoid system with cannabinoid receptor agonists: pharmacological strategies and therapeutic possibilities. Philos Trans $R$ Soc Lond B Biol Sci 367:3353-3363.

Piro JR, Benjamin DI, Duerr JM, Pi Y, Gonzales C, Wood KM, Schwartz JW, Nomura DK, and Samad TA (2012) A dysregulated endocannabinoid-eicosanoid network supports pathogenesis in a mouse model of Alzheimer's disease. Cell Rep 1: 617-623.

Salvado MD, Alfranca A, Escolano A, Haeggström JZ, and Redondo JM (2009) COX-2 limits prostanoid production in activated HUVECs and is a source of PGH2 for transcellular metabolism to PGE2 by tumor cells. Arterioscler Thromb Vasc Biol 29:1131-1137.

Schlosburg JE, Blankman JL, Long JZ, Nomura DK, Pan B, Kinsey SG, Nguyen PT, Ramesh D, Booker L, Burston JJ, et al. (2010) Chronic monoacylglycerol lipase blockade causes functional antagonism of the endocannabinoid system. Nat Neurosci 13:1113-1119.

Simon GM and Cravatt BF (2010) Activity-based proteomics of enzyme superfamilies: serine hydrolases as a case study. J Biol Chem 285:11051-11055.

Singh J, Petter RC, Baillie TA, and Whitty A (2011) The resurgence of covalent drugs. Nat Rev Drug Discov 10:307-317.

Sugaya Y, Yamazaki M, Uchigashima M, Kobayashi K, Watanabe M, Sakimura K, and Kano M (2016) Crucial roles of the endocannabinoid 2-arachidonoylglycerol in the suppression of epileptic seizures. Cell Rep 16:1405-1415.

Sugiura T, Kondo S, Sukagawa A, Nakane S, Shinoda A, Itoh K, Yamashita A, and Waku K (1995) 2-Arachidonoylglycerol: a possible endogenous cannabinoid receptor ligand in brain. Biochem Biophys Res Commun 215:89-97.

Terrone G, Pauletti A, Salamone A, Rizzi M, Villa BR, Porcu L, Sheehan MJ, Guilmette E, Butler CR, Piro JR, et al. (2018) Inhibition of monoacylglycerol lipase terminates diazepam-resistant status epilepticus in mice and its effects are potentiated by a ketogenic diet. Epilepsia 59:79-91.

Tjølsen A, Berge OG, Hunskaar S, Rosland JH, and Hole K (1992) The formalin test: an evaluation of the method. Pain 51:5-17.

Tosun NC, Gunduz O, and Ulugol A (2015) Attenuation of serotonin-induced itch responses by inhibition of endocannabinoid degradative enzymes, fatty acid amide hydrolase and monoacylglycerol lipase. J Neural Transm (Vienna) 122:363-367.

Viader A, Blankman JL, Zhong P, Liu X, Schlosburg JE, Joslyn CM, Liu QS, Tomarchio AJ, Lichtman AH, Selley DE, et al. (2015) Metabolic interplay between astrocytes and neurons regulates endocannabinoid action. Cell Rep 12:798-808.

von Rüden EL, Bogdanovic RM, Wotjak CT, and Potschka H (2015) Inhibition of monoacylglycerol lipase mediates a cannabinoid 1-receptor dependent delay of kindling progression in mice. Neurobiol Dis 77:238-245.

Warner TD, Giuliano F, Vojnovic I, Bukasa A, Mitchell JA, and Vane JR (1999) Nonsteroid drug selectivities for cyclo-oxygenase-1 rather than cyclo-oxygenase-2 are associated with human gastrointestinal toxicity: a full in vitro analysis. Proc Natl Acad Sci USA 96:7563-7568.

Weerapana E, Wang C, Simon GM, Richter F, Khare S, Dillon MB, Bachovchin DA, Mowen K, Baker D, and Cravatt BF (2010) Quantitative reactivity profiling predicts functional cysteines in proteomes. Nature 468:790-795.

Wilkerson JL, Niphakis MJ, Grim TW, Mustafa MA, Abdullah RA, Poklis JL, Dewey WL, Akbarali H, Banks ML, Wise LE, et al. (2016) The selective monoacylglycerol lipase inhibitor MJN110 produces opioid-sparing effects in a mouse neuropathic pain model. J Pharmacol Exp Ther 357:145-156.

Xu T, Park SK, Venable JD, Wohlschlegel JA, Diedrich JK, Cociorva D, Lu B, Liao L, Hewel J, Han X, et al. (2015) ProLuCID: an improved SEQUEST-like algorithm with enhanced sensitivity and specificity. J Proteomics 129:16-24.

Yesilyurt O, Cayirli M, Sakin YS, Seyrek M, Akar A, and Dogrul A (2016) Systemic and spinal administration of FAAH, MAGL inhibitors and dual FAAH/MAGL inhibitors produce antipruritic effect in mice. Arch Dermatol Res 308:335-345.

Zhang J and Chen C (2018) Alleviation of neuropathology by inhibition of monoacylglycerol lipase in APP transgenic mice lacking CB2 receptors. Mol Neurobiol $\mathbf{5 5 : 4 8 0 2 - 4 8 1 0}$

Zhang J, Teng Z, Song Y, Hu M, and Chen C (2015) Inhibition of monoacylglycerol lipase prevents chronic traumatic encephalopathy-like neuropathology in a mouse model of repetitive mild closed head injury. J Cereb Blood Flow Metab 35:443-453.

Address correspondence to: Dr. Jacqueline L. Blankman, Abide Therapeutics, 10835 Road to the Cure, Suite 250, San Diego, CA 92121. E-mail: jackie@ abidetx.com 